\title{
Sugars: burden or biomaterials of the future? $\uparrow$
}

\author{
Iva Pashkuleva ${ }^{* a b}$ and Rui L. Reis ${ }^{a b}$ \\ Received 26th May 2010, Accepted 30th July 2010 \\ DOI: 10.1039/c0jm01605e
}

During the past few years, the field of tissue engineering (TE) has been shifting from replacement to regenerative strategies. Following this tendency, the requirements for biomaterials to be used in TE have been also changing. While a few decades ago bioinert materials that do not provoke undesired body responses were in the focus of material sciences, nowadays third generation biomaterials mimicking the nanoscale mechanisms of the interactions between cells and their in vivo environment are the target of material design. Although these mechanisms involve different bioactive molecules, until now mainly strategies involving small peptide epitopes that copycat specific sequences of complex proteins have been exploited. The breakthroughs that such approaches brought to biomaterials and TE fields are undeniable. Nevertheless, the important role that carbohydrates play in tissue structuring and function is still poorly explored and exploited in this context and we believe that this is one of the missing pieces in the TE puzzle. Carbohydrates are an integral part of our life. We are literally covered by them: from bacteria to mammalian cells, the molecular landscape of the cell surface is coated with sugars forming the so-called glycocalyx. This strategic placement of the sugars makes them crucial for the development, growth, function and/or survival of an organism. It is believed that the structural diversity of carbohydrates is the key for understanding and controlling those processes because of the huge number of ligand structures, which sugars can display in molecular recognition systems. However, their main advantages: the intricacy and the large natural diversity have turned against the scientists and have hampered their study. As a result, the field of glycomics is much less developed compared to its counterparts genomics and proteomics within TE. Recent advances in carbohydrate synthesis, sensing technologies and processing methodologies are inducing rapid changes in this field and will be discussed in this paper. The use of carbohydrates as an interrogation and modulation tool for better understanding of the complexity and dynamics of the natural three-dimensional environment of the cells will be also focussed.

\section{Introduction}

The field of tissue engineering (TE) exploits living cells and biomaterials in a variety of ways to restore, maintain, or enhance tissues and organs. ${ }^{1}$ During the 1960s and 1970s, it was believed that the biomaterials used must be bioinert and thus minimising the body's response to foreign devices. ${ }^{2}$ About ten years later, this view was changed and second generation ${ }^{3}$ bioactive materials that elicit a controlled action and reaction in a physiological environment had emerged. With the development of nanobiotechnology, the researchers have learnt from Nature about the structure and properties of living tissues and systems. Nowadays, it is well known that cells can sense, integrate and respond to environmental cues. This understanding brought the third generation biomaterials ${ }^{4}$ able to cross-talk with cells and biomolecules from the physiological milieu and thus stimulating

a3B's Research Group - Biomaterials, Biodegradables and Biomimetics, University of Minho, Headquarters of the European Institute of Excellence on Tissue Engineering and Regenerative Medicine, AvePark, 4806-909 Taipas, Guimarães, Portugal. E-mail: pashkuleva@dep.uminho. $p t$

${ }^{b} I B B$ - Institute for Biotechnology and Bioengineering, PT Government Associated Laboratory, Braga, Portugal

$\dagger$ This paper is part of a joint Journal of Materials Chemistry and Soft Matter themed issue on Tissue Engineering. Guest editors: Molly Stevens and Ali Khademhosseini. specific cellular responses at the molecular level. The strategic placement of oligosaccharides and glycoconjugates on the cell surface makes them a key element of this communication process. However, for many years the chemistry and biology of carbohydrates have been considered as a Cinderella field ${ }^{5}$ : an area that involves tremendous work but stays in the shadow of her cousins genomics and proteomics. Initially, carbohydrates were only seen as structural components of natural products or as a source of energy. Only in the late 1980s, the field of glycobiology emerged as an attitude integrating the traditional carbohydrate chemistry and biochemistry with a modern understanding of the cell and molecular biology of glycans and their conjugates with proteins and lipids. ${ }^{6,7}$ Hence, carbohydrate research has become a hot subject only after the scientists understood that the functions of cells and organisms cannot be explained by protein and nucleic acids alone. Once viewed as a hydrophilic heterogeneous nuisance to be removed from the cell surface or secreted proteins of interest, oligosaccharides have been recognised as an intricate network encoding a wealth of information. Nowadays, their key participation in the intercellular communication, various recognition processes and signal transduction events (Fig. 1) is confirmed. However, the precise mechanism of many of those processes remains to be elucidated and though potential opportunities exist, the pace of development of carbohydrate research has been slower than the ones of 


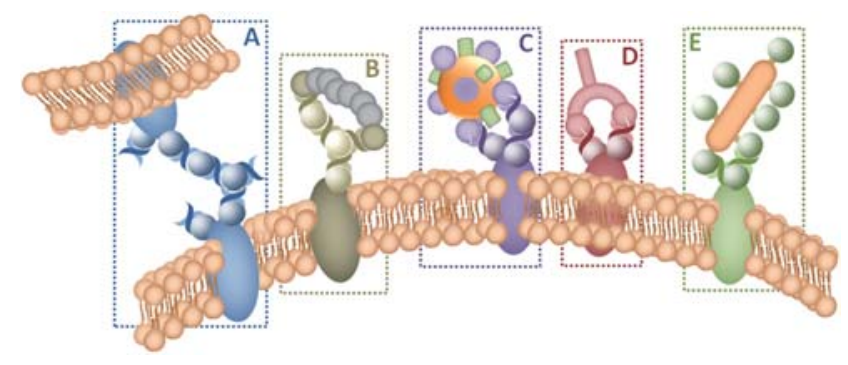

Fig. 1 Participation of cell surface carbohydrates in recognition events with another cell (A), toxins (B), viruses (C), antibodies (D) and bacteria (E).

proteins and nucleic acids. This embarrassing fact is in large part due to their inherent structural complexity, the great difficulty in determining their sequences, and the fact that their biosynthesis could not be predicted directly from a DNA template, i.e. their genetic manipulation is far more complicated than for proteins and nucleic acids.

In 2003, glycomics, the systematic effort to understand the biosynthesis, structure and function of carbohydrate polymers and oligosaccharides, was included ${ }^{8}$ in the list of the 10 emerging technologies that will change the world. In fact, this past decade was marked by tremendous breakthroughs in this field: we have witnessed the development of the first automatic carbohydrate synthesis and the first trials with and on carbohydrate arrays. These recent advances have provided critical material for biological investigations, which resulted in discoveries of new bioactive carbohydrates and revealed the great potential of oligosaccharides in the field of TE and as therapeutics preventing virus and bacterial infections or retarding cancer progress.

\section{The complexity of carbohydrates}

The complexity of carbohydrates has been attracting the attention of researchers from different scientific fields for more than a century (Table 1). Since Emil Fischer determined the stereochemistry of their building blocks, the monosaccharides, up to today scientists are seeking to understand the Nature's choice of certain carbohydrates and her ways of producing, manipulating and employing them.

What makes carbohydrates that unique and what distinguishes them from the other two classes of biomacromolecules, proteins and polynucleotides, responsible for the information transfer in the biological systems? Unlike proteins and polynucleotides, saccharides are not direct genetic products. Instead, they are synthesised by complex pathways, regulated by multiple factors including metabolic levels of sugar nucleotides, expression and localisation of glycosylation enzymes, and protein-trafficking mechanisms. As a result, carbohydrates differ from other biological macromolecules in two important characteristics: they can be highly branched molecules and many different linkages can bind their residues. For example, any pair of six-carbon monosaccharides can be linked in 11 different ways (Fig. 2).

Assuming that ten distinct types of monosaccharides are commonly available in mammalian tissues ${ }^{9}$ (Table 2), the formation of $10 \times 10 \times 11$, or 1100 different disaccharides is theoretically possible. In comparison, two amino acids can be joined in only one way, creating $20^{2}$ dimers and the possible combinations for the nucleotides are only $4^{2}$ or 16 dinucleotides. ${ }^{9-11}$ The number of possible combinations rapidly increases with each additional monosaccharide and outpaces those for the respective peptides and oligonucleotides. Usually, saccharidebinding proteins recognise hexaoligomer or smaller oligosaccharides. Based on this fact and on seven major structural features of carbohydrates: (i) epimers, including D and L forms; (ii) linear sequence of core and linear branches; (iii) ring size; (iv) anomeric configuration; (v) linkage position; (vi) branching positions and (vii) reducing terminal attachment, Laine calculated $^{12}$ that the theoretical number of all possible linear and branched isomers of a hexasaccharides is $>1.05 \times 10^{12}$. Therefore, in a short sequence of carbohydrates can potentially display a huge number of ligand structures to the binding sites of proteins in molecular recognition systems. Thus, their potential to encode information in a short sequence is several orders of magnitude higher than that of any other biological macromolecule. ${ }^{11}$ Because of this potential, carbohydrates are called the third alphabet ${ }^{13}$ of the language of life (behind nucleic acids and proteins): they are like the accents on spoken words - they change the meaning without changing the spelling!

Moreover, we must consider two other issues when speaking about the complexity of the carbohydrates: (i) in a monosaccharide unit, some or all of the hydroxyl groups can be replaced by phosphonic or sulfonic groups (e.g. glycosaminoglycans) and (ii) in a cell organism, the oligosaccharides are usually attached to another molecule forming so-called glycoconjugates, which include glycoproteins, glycolipids and glycosylphosphatidylinositol (GPI) anchors. Given this potential structural diversity of carbohydrates, how complex is the glycome really? In fact, the number of structural combinations encountered in nature has not yet been elucidated. A recent report by Seeberger et $a .^{9}{ }^{9}$ focuses the analysis of structural complexity of mammalian oligosaccharides actually found in nature (carbohydrates database). This report demonstrated that a rather limited set of the theoretically possible building blocks is sufficient to access the majority of the mammalian glycospace; just 11 monosaccharide connections account for $>75 \%$ of all linkages and only 36 building blocks are required to construct $75 \%$ of the studied 3299 mammalian oligosaccharides. Unfortunately for the glycoscientists, the bacteria glycome is much complex, composed by many more building blocks that are connected in a dazzling array of branched structures. ${ }^{14}$ So far, it has not been shown how and why the Nature chooses these blocks. These questions have been addressed for many years but they are still difficult to be answered since the great majority of glycans in nature are yet to be discovered and structurally defined.

\section{Biological roles of carbohydrates}

For the past decades, a variety of theories have been presented regarding the biological roles of the carbohydrates. ${ }^{7,15,16}$ It appears that glycans span a large spectrum of bioactivities: from those that are trivial such as source of energy or pure structural role to those that are crucial for the development, growth, function or survival of an organism. This variety is not surprising if one keeps in mind their structural diversity. Moreover, 
Table 1 Some of the most important advances in the elucidation of the complexity of carbohydrates. A detailed table is available elsewhere ${ }^{7}$

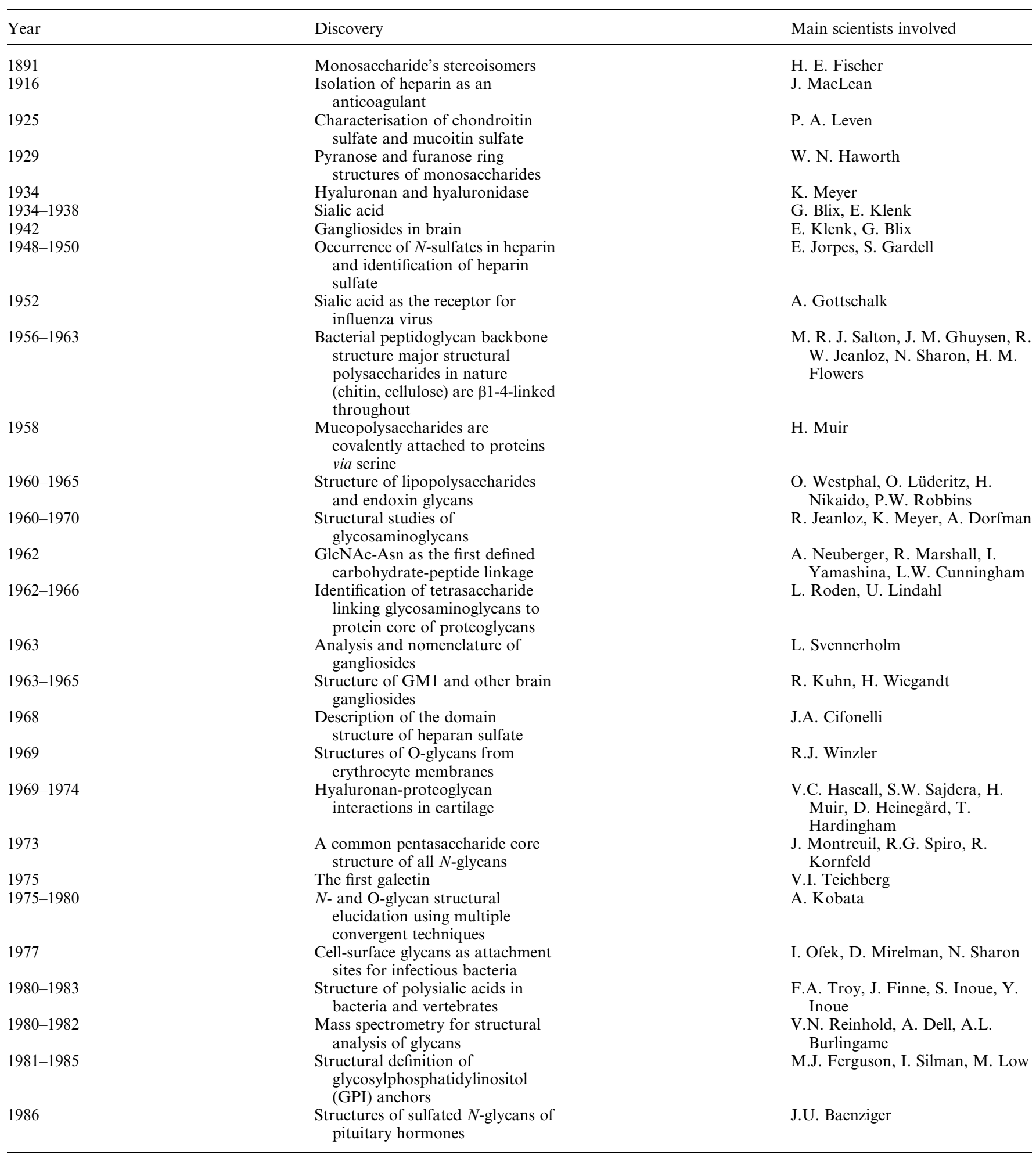

carbohydrates have very strategic location on the cell surface (Fig. 3). From bacteria to mammalian cells, the molecular landscape of the cell surface is coated with sugars (so-called glycocalyx), which makes them the first points of contact between cells and their environment. The sugars placed on the cell surface can either build a protective layer from harmful physical forces or regulate interactions of cells with the environment. Thus, they are in a position to modulate or mediate a plethora of biological processes in cell-cell, cell-matrix, and cell-molecule interactions. Therefore they have a great potential in the TE field as bioactive agents able to control the development and function of a complex multicellular organism. ${ }^{17}$ The 


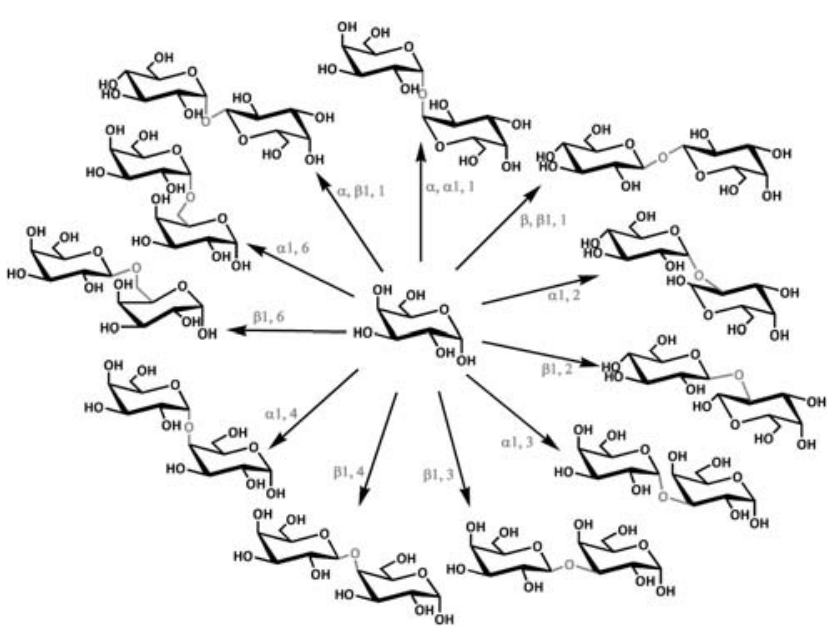

Fig. 2 Possible linkages between two identical monosaccharides.

mediation of the above mentioned processes can be achieved via different ways ${ }^{\mathbf{1 8}, 19}$ : some of the sugars act as classical ligands or co-receptors that facilitate cell attachment and mediate signalling (e.g. glycosaminoglycans of the mammalian cell surface serve as co-receptors for proteins that affect the respective cell, while others act indirectly by regulating interactions of molecules via steric hindrance, exclusion or recruitment. The flexible nature of the sugars in a glycoprotein allows for many more confirmation than would their purely protein amino-acid counterparts. Thus, sugars can promote proper folding of proteins by stabilising particular conformation and in some cases protecting the protein portion of the glycoprotein against degradative enzymes.

Alterations in cell-surface glycans are commonly observed in a wide variety of diseases and are strongly correlated with cancer prognosis, metastatic potential and the organ specificity of metastasis. Moreover, strong evidence that glycan remodelling is an integral part of disease progression has led to speculation that carbohydrates are key diagnostic and prognostic indicators as well as therapeutic targets of interest. Cell surface molecules are also strategically exposed for surveillance by the immune system allowing for the potential immune recognition of abnormal cells. Despite those potential abilities of carbohydrates, the lack of tools for studying glycobiology prevented biologists and medical researchers from addressing research problems that involve them until very recently. ${ }^{20}$ Defining the function of glycans in the biological milieu presents a challenge due to the complexity of carbohydrates at the structural, biosynthetic and functional levels.

\section{Synthesis of oligosaccharides}

Access to structurally defined carbohydrates is a prerequisite for revealing their function and establishment of the structural features important for this function. Carbohydrate biosynthesis and diversification involves several complex steps. ${ }^{21}$ The assembling takes place in the endoplasmic reticulum and Golgi apparatus and it affords significant product microheterogeneity (Fig. 3), which explains the difficulties in obtaining homogeneous and chemically defined glycoconjugates from natural sources. ${ }^{16,22}$ On the other hand, carbohydrates are also challenging for the synthetic chemists because of the many functional groups that have to be protected in order to get one, specific group to react. Additional complication arises from the need to control the stereochemistry of the newly formed glycosidic linkage (Fig. 2).

Generally, the strategies for synthesis of carbohydrates can be divided into chemical and enzymatic ones. The chemical approaches are formidable; typically they involve a large number of organic reactions aiming at stereo- and regiochemical control of the forming glycosidic linkage followed by time consuming and expensive purification steps. ${ }^{23}$ Chemical strategies include traditional solution-phase chemical synthesis, one-pot reactivity based glycosylations and automated solid-phase synthesis. For all those strategies, the preparation of selectively protected monosaccharide units is a crucial step. These units should be designed to present a strategically positioned free hydroxyl group (a nucleophilic acceptor) or a labile leaving group at the anomeric carbon that acts as a glycosyl donor. Thus, the main advances in different chemical approaches are related with the unveiling of anomeric groups that allow for high-yields, selective and reliable formation of different glycosidic linkages (e.g. Fig. 4).

The one-pot glycosylation method refers to an approach in which several glycosyl donors are allowed to react sequentially in the same vessel resulting in a single oligosaccharide product. The method was proposed by Raghavan and Kahne in $1993^{25}$ when the synthesis of $\alpha$-linked deoxytrisaccharide by sequential chemoselective activation of glycosylsulfoxide and thioglycoside using a single activator was described. Lately, numerous research groups have been working on the various one-pot synthetic strategies and a significant result of these efforts has been the development of programmable one-pot synthesis. ${ }^{26,27}$ The underlying principle of one-pot glycosylation is the ability to control glycosyl donor reactivity. Over the years three major concepts have emerged as main ones in this field (Fig. 5): (i) chemoselective strategy; (ii) orthogonal glycosylation and (iii) pre-activation strategy. ${ }^{28}$

In the chemoselective strategy (Fig. 5A), the protecting groups are used as a tool for tailoring the reactivity of glycosyl donors: the leaving groups in the glycosylation reaction are activated by electron-donating groups (arm) and deactivated by electronwithdrawing groups (disarm). The viability of this so-called armed-disarmed concept was firstly documented by Paulsen ${ }^{29}$ and later demonstrated by Fraser-Reid et al. ${ }^{30}$ The majority of the following studies have been qualitative until Lay's group initiated a breakthrough study ${ }^{31,32}$ aiming at the estimation of relative reactivity values (RRV) of some gycosyl donors by NMR spectroscopy. The RRVs describe the product ratio when two donors compete for a single acceptor. Therefore, they can be used to predict the product when multiple glycosyl donors are present and/or to select a proper donor. An alternative route for determination of RRVs was proposed by Wong et $a .^{26} \mathrm{In}$ their study, they designed competition experiments with various thioglycoside-activated glycosyl donors and donor-acceptors. The RRVs in this case were estimated by HPLC. Based on the created reactivity database, the OptiMer software was created which allows search and selection of suitable building blocks for one-pot assembly of a desired oligosaccharides or a library of individual oligosaccharides. 
Table 2 The 10 most abundant monosaccharides (sugar "letters") found in mammalian oligosaccharides (data are based on the results published elsewhere ${ }^{9}$ ). While nucleic and amino acids can be represented with one letter in the polynucleotides and proteins, the complexity of sugars does not allow this representation and instead colour symbols are typically used by the glycobiologists

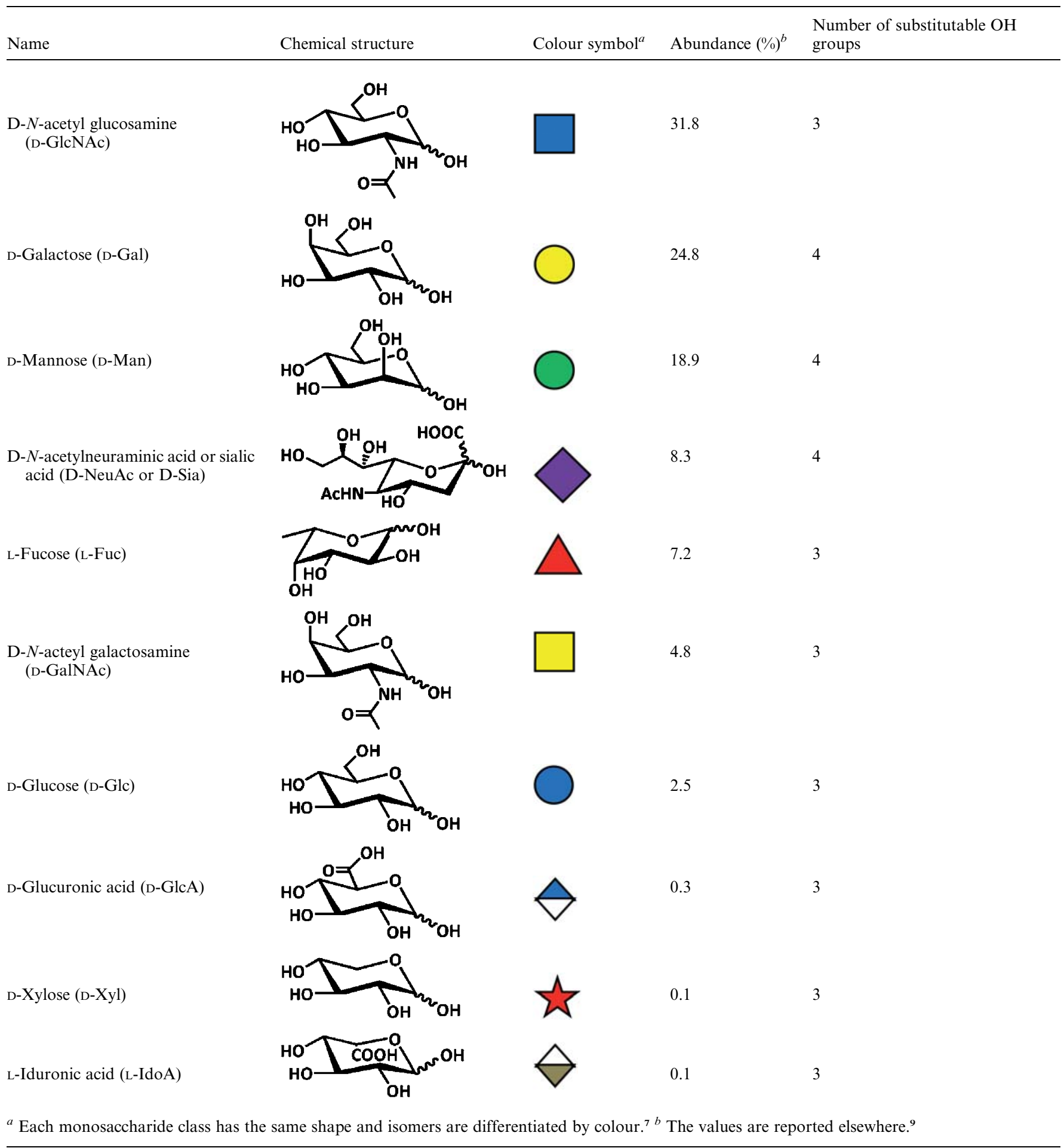

Besides changing the protective groups, other variables such as the nature of the anomeric leaving groups or the effect of the solvent on the donors' reactivity during the glycosylation process can be also used to facilitate efficient oligosaccharide one-pot synthesis. Thus, the orthogonal strategy (Fig. 5B) introduced by Takahashi et al. $^{33,34}$ uses various anomeric leaving groups to tailor the reactivity of the glycosyl donors. Compared with the chemoselective glycosylation, this strategy has an advantage: it allows the condensation of building blocks independently of their RRVs. In 2000 Takahashi's group reported ${ }^{27}$ the first multistep synthesis performed in a parallel manual synthesiser (Quest 210). The authors described the synthesis of a library of 54 linear and 


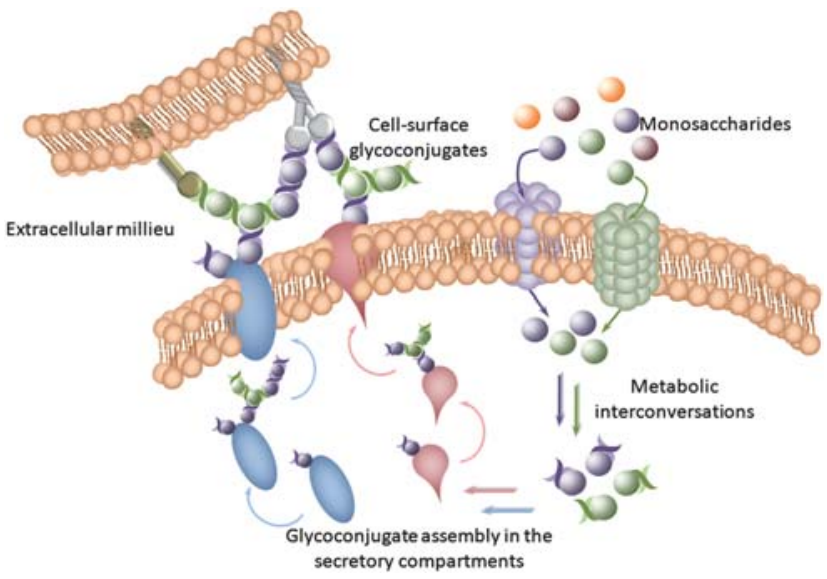

Fig. 3 A schematic presentation of glycoconjugate biosynthesis, surface expression and recognition. Only few polysaccharides are found inside cell, while most oligo- and polysaccharides are part of the extracellular matrix or are present on the cell surface where they either play a protective role or regulate the interactions of cells with the environment.

18 branched trisaccharides, which later was extended to heptasaccharides. ${ }^{35}$ In fact, this approach can be considered as an initial form of the automatic oligosaccharide synthesis.

The examples given above demonstrate that reactivity-based one-pot synthesis has greatly facilitated the glycosylation process. However, the excessive synthetic work required to obtain building blocks with suitable anomeric reactivities complicates the overall procedure and decreases its efficiency. In 2004, Huang et al. proposed $^{36}$ an alternative approach of iterative one-pot synthesis of oligosaccharides based on the pre-activation strategy (Fig. 5C). This strategy integrates the advantages of both chemoselective strategy (activation under a single set of glycosylation conditions) and orthogonal strategy (independence of RRVs). The reaction scheme involves pre-activation of glycosyl donors, which generates a reactive intermediate in the absence of acceptor. After addition of the second building block to the pre-activated donor, a disaccharide is formed which can be activated in the same manner. The process can be repeated several times in the same flask until the targeted oligosaccharide is obtained. Several requirements need to be fulfilled in order to have an efficient reaction ${ }^{28,37}$ : (i) the activator should work with a wide range of glycosyl donors in a stoichiometric amount and to be completely consumed by the donor in order to prevent further activation of the following building blocks; (ii) the generated intermediate must be sufficiently long-living to allow the addition of the acceptor but yet reactive enough for a high-yielding glycosyl coupling; (iii) side products formed during the reaction should not interfere with the glycosylation process. The obstructions associated with the applicability of the pre-activation strategy to the synthesis of complex oligosaccharides are related with the breach of some of these requirements. In some cases the preactivated donors do not react with the acceptors and thus, an optimisation of the coupling conditions or/and development of more powerful activators are required. Another obstacle is related with a side reaction: a regeneration phenomenon has been observed occasionally when thioglycosides bear the same leaving group. This effect can be a result from aglycon transfer and there are several studies devoted to the resolution of this problem. ${ }^{38-40}$

A

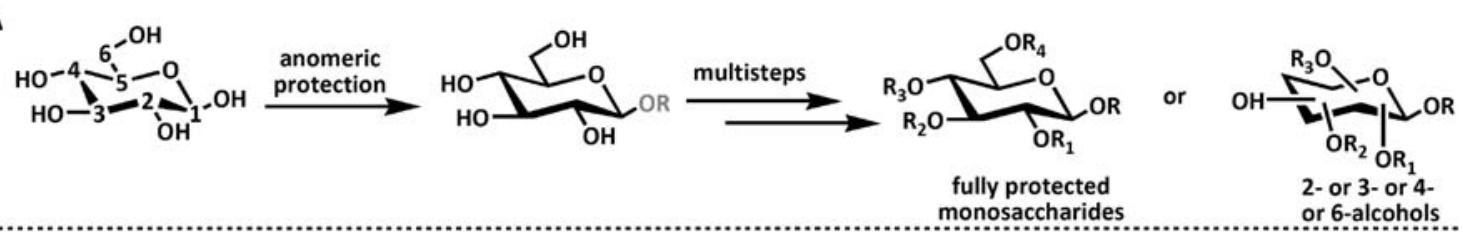

B
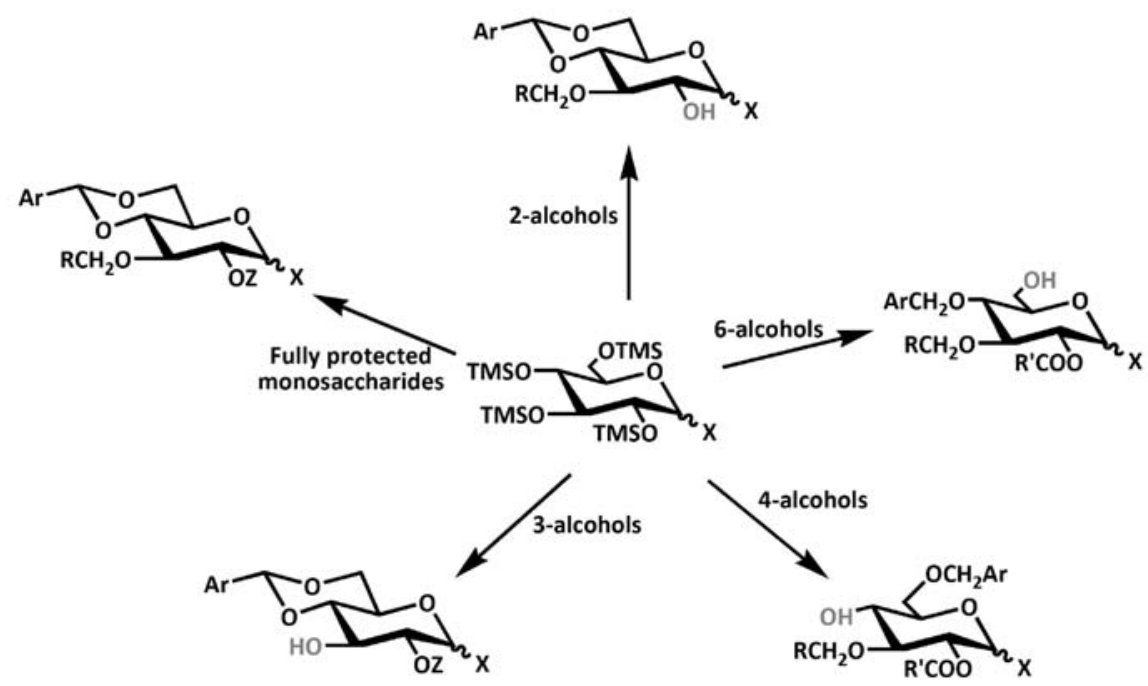

Fig. 4 Regioselective protection of hydroxyl groups in monosaccharides: traditional multistep approach (A) and one-pot approach proposed by Hung et $a l^{24}$ (B, OTMS = O-trimethylsilyl group) which allows synthesis of 152 different glucose building blocks by only four different types of one-pot reactions. 

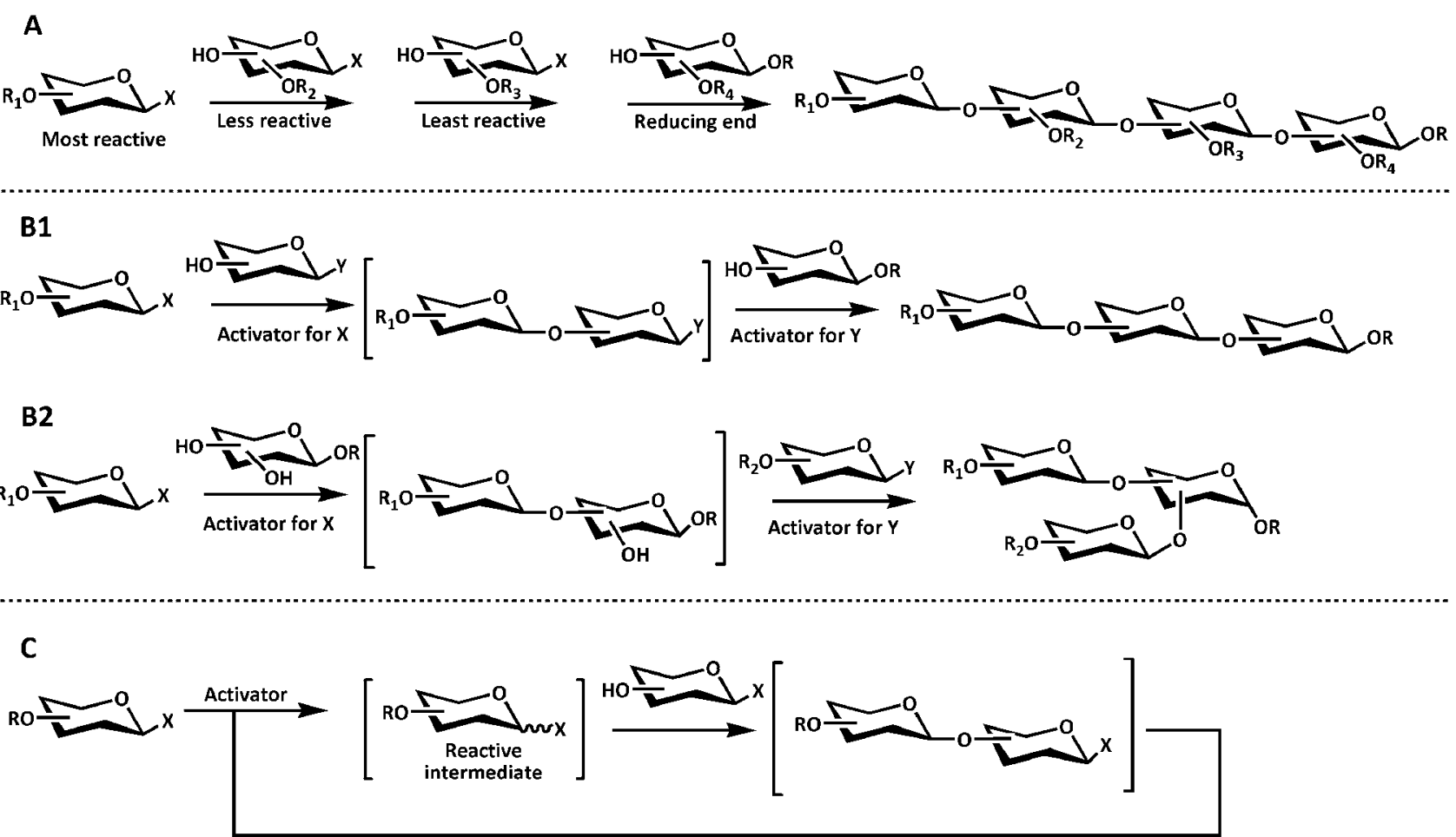

Fig. 5 Concepts for one-pot glycosylation: chemoselective strategy (A); orthogonal strategy for linear (B1) and branched (B2) oligosaccharide synthesis; and glycosylation based on the pre-activation strategy $(\mathrm{C})$.

Undoubtedly, the automated solid-phase synthesis of oligosaccharides developed and proposed by Seeberger's group ${ }^{41}$ is one of the milestones in the rapidly developing field of glycomics. The impact of an automated oligosaccharide synthesiser can be easily understood if one analyses the breakthroughs, which automated peptide and oligonucleotide synthesis brought in the field of TE, biochemistry and associated biomedical applications.
The principle is the same (Fig. 6): the growing chain of an oligosaccharide is covalently linked to an insoluble support such as Merrifield's resin. Reagents are added to a suspension of the solid support and allowed to react with or modify the reactant attached to the support. An excess of reagents ensures the completion of the reaction. Once the synthesis sequence is terminated, all unreacted reagents or side products remaining in the

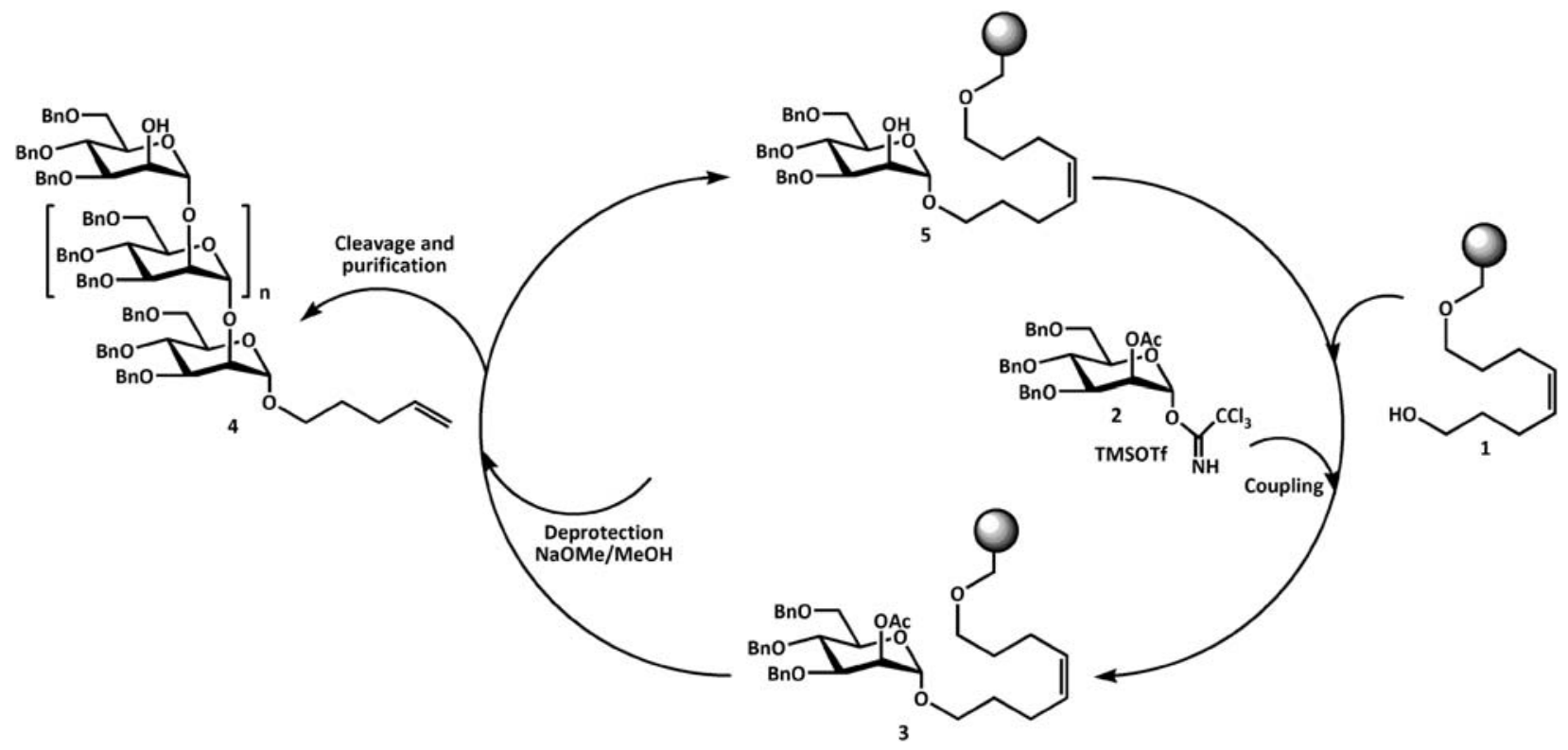

Fig. 6 Automated oligosaccharides synthesis showing coupling, deprotection and final detachment from the solid support employed for the synthesis of an oligo- $\alpha$-mannoside bearing a pentenyl linker on the reducing end. 
solution can be removed by simple filtration and washing. The targeted product is collected after cleavage from the solid support. As in the other synthetic strategies, the development and use of protecting groups is pivotal for the success of the synthetic procedure. Permanent protecting groups, such as benzyl ethers (BnO-) are used in the positions where a free hydroxyl will be present in the final product. Temporary protecting groups, such as esters $\left(\mathrm{CH}_{3} \mathrm{COO}-\right.$, AcO-) are used to mask hydroxyls that will participate in the glycosylation. Besides the use of orthogonal protective groups to assist controlled regioselectivity and thus controlled branching of the carbohydrate chain, the stereochemistry of the anomeric carbon must be also controlled. Placement of participating protective groups at the C2 hydroxyl or amine groups ensures the formation of transglycosidic linkage, whereas non-participating groups are needed for the preferential installation of $c i s$-glycosides. ${ }^{20}$ Another key issue in the solid-phase synthesis is the choice of the linker to the resin: it must remain stable during the coupling and deprotection events but to be readily cleavable following the synthesis and ideally to leave a spacer on the reducing end for further functionalisation. ${ }^{42}$ In their works, Seeberger et al. have employed 4-octenediol linker (Fig. 6,1) that can be cleaved via olefin crossmetathesis in the presence of Grubbs catalyst and ethylene. ${ }^{41}$

As in the strategies described above, the automated oligosaccharide synthesis has its advantages and remaining challenges. ${ }^{20,42}$ One of its main advantages is shortening of the time required for the synthesis; in about one day the process is complete compared to manual synthesis, which can take days and the final yield is much lower. Some of the problems associated with the widespread use of this method are: (i) the needed excess of building blocks that are not commercially available and their procurement constitutes the biggest time commitment for carbohydrate assembly; (ii) difficult incorporation of certain monosaccharides such as sialic acid; (iii) the cross-metathesis reaction used to cleave the linker in many cases takes time as long as the assembly process itself; (iv) the protective groups removal usually requires several steps and good chemical skill.

Enzyme catalysed synthesis of oligosaccharides ${ }^{43,44}$ is often used as an alternative of the existing chemical methods. The main advantage of enzymatic synthesis is that substrates are used in their natural form, i.e. no protecting groups are required to direct the regio- and stereo-specificity of the glycosidic bond formation. Two types of natural enzymes that can be used in this process are available, namely glycosyltransferases and glycosidases (Fig. 7).

Complex glycoconjugates such as glycopeptides, glycoproteins and glycolipids have been synthesised through multiple-enzyme processes with the help of enzymes as sulfotransferases, proteases, lipases and aldolases (e.g. Table 3).

The use of enzyme-based synthesis implicates several restrictions. ${ }^{37,43-45}$ As already mentioned, glycosyltransferases are not always readily available and thus expensive nucleotide-activated glycosides are required. Glycosyltransferases from microbial sources are a viable alternative as it has been demonstrated by the synthesis of a range of oligosaccharide analogues. ${ }^{46,47}$ Glycosidases are another alternative. In comparison with glycosyltransferases, they are inexpensive, stable and readily available.
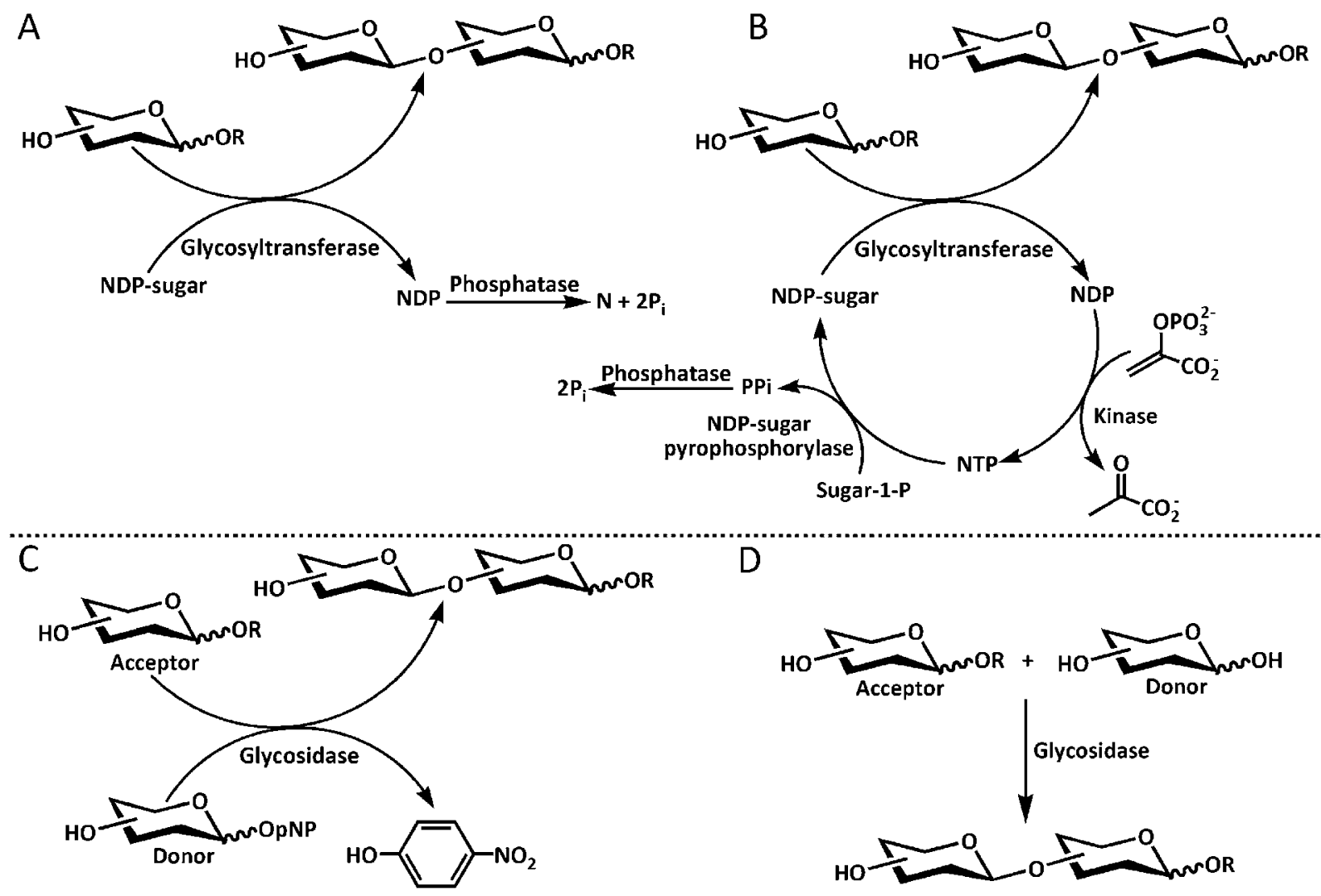

Fig. 7 General protocols for glycosyltransferase (A,B) and glycosidase (C,D) based synthesis: addition of phosphatase (A); recycling of sugar nucleotides (B); trans glycosylation (C) and reverse hydrolysis (D). 
Table 3 Enzymes commonly used in glycoconjugates synthesis

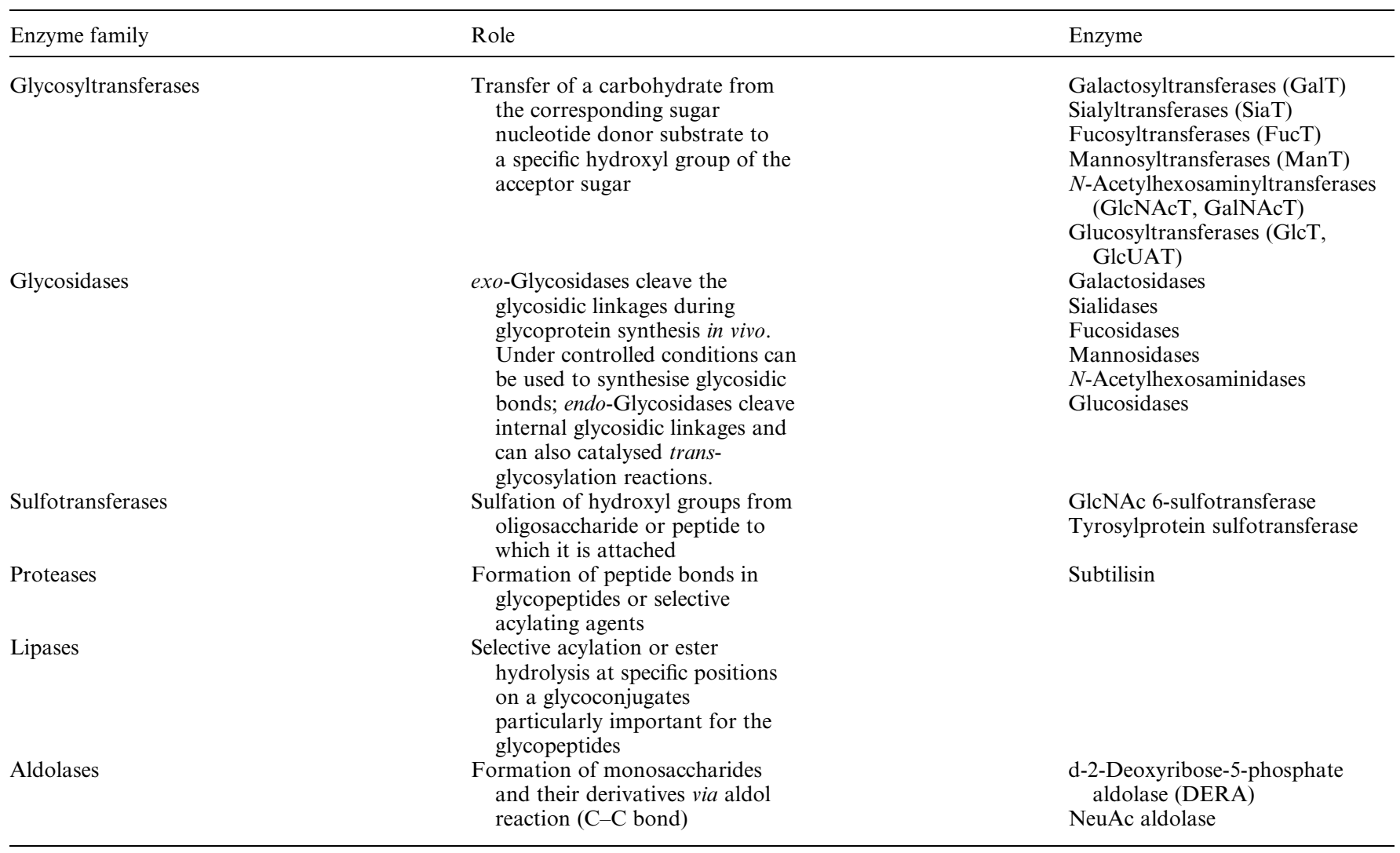

Moreover, they use cheaper substrates, such as sugar halides and $p$-nitrophenyl glycosides. Their main drawbacks are: poor regiospecificity that can result in the formation of multiple products; the typical lower yields owing to the challenge of driving reactions in a thermodynamically unfavourable direction and because of enzymatic degradation of the reaction products. However, the Withers group found ${ }^{48,49}$ that mutagenesis of glycosidases (replacement of a nucleophilic aspartic or glutamic acid with another amino acid) produces an enzyme named as "glycosynthase" that can catalyse the synthesis of a saccharide from a fluorosugar donor but does not catalyse the hydrolysis of the resulting product. Although multi-enzymatic methods have been developed and proved to be useful for a large-scale process (Fig. 8), the identification of other enzymes with high activity and broad substrate specificity remains a challenge in the synthesis of glycans.

\section{Carbohydrate arrays}

A major challenge in TE is to define the interactions between cellcoating sugars and different bioentities available in the extracellular environment such as proteins, enzymes, RNA, cells, pathogens and to understand how they recognise each other. ${ }^{51-53}$ A better understanding of these interactions would greatly aid to the elucidation of different signalling pathways and thus, eventually lead to faster regeneration process as well as to improved diagnostic and therapeutic tools. However, this is not an easy task since the carbohydrate-bioentity interactions are often weak (e.g. the affinity of GBPs for their glycan ligand is typically with
$\left.K_{\mathrm{d}}=1-1000 \mu \mathrm{M}^{53}\right)$. Additional complications arise from the fact that efficient binding relies on multivalent interactions (cluster effect) that are very difficult to measure experimentally. ${ }^{54}$ Moreover, carbohydrate heterogeneity and the cross-reactivity of sugar binding proteins require large number of carbohydrate ligands to be screened. ${ }^{18,19}$ Microarray techniques offer the

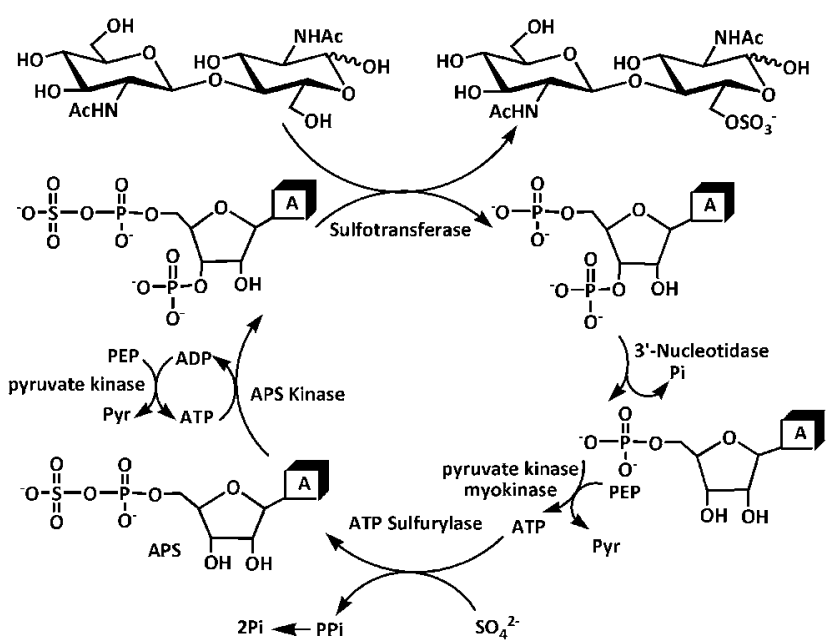

Fig. 8 Multiple enzyme system used in the large-scale synthesis of $\mathrm{SLe}^{\mathrm{x}}$. The method has been extended to the synthesis of hyaluronic acid and it is believed that all the oligosaccharides in mammalian systems can be prepared by this methodology. ${ }^{50}$ 
possibility of high-throughput analysis of these interactions using minimal quantities of sugar samples. In order to provide both satisfying selectivity and quantitative performance, the carbohydrate arrays must fulfil several requirements $\mathrm{s}^{55}$ : (i) carbohydrates must be present in a regular and homogeneous manner so that all immobilised ligands have equal activity toward the analytes present in the solution; (ii) the density of the immobilised carbohydrates must be very well controlled because many carbohydrate-analyte interactions are polyvalent in nature and their affinity is extremely sensitive to the density and orientation of individual carbohydrates ${ }^{19}$; (iii) a spacer between the carbohydrate and the surfaces is needed to provide the analyte with access to the immobilised sugar; (iv) from practical point of view it is very important that the array is compatible with several detection methods (e.g. fluorescence, surface plasmon resonance, quartz crystal microbalance, etc.).

The first trials on and with carbohydrate arrays were inspired from the protein, gene and cell chips. Several approaches for polysaccharides and glycoconjugates microarrays ${ }^{56,57}$ using natural and synthetic oligosaccharides ${ }^{58-60}$ and monosaccharides ${ }^{55,59-62}$ were reported in 2002 by different groups. Since then intensive research has been carried out on different strategies for immobilisation of natural and synthetic sugars on glass, plastic and gold surfaces (Table 4). So far, no particular fabrication method has emerged as a winning approach and therefore, new methods to array carbohydrates are still under development.

The reported approaches differ primarily in the method of attachment of carbohydrate to the solid support. So far, four general strategies (Fig. 9) have been communicated in the literature: (i) non-specific, non-covalent immobilisation of unconjugated carbohydrates on unmodified surfaces; (ii) site-specific, non-covalent immobilisation of conjugated carbohydrates on unmodified surfaces; (iii) site-specific, covalent immobilisation of conjugated carbohydrates on derivatised surfaces; and (iv) sitespecific, covalent immobilisation of chemically unmodified carbohydrates on modified surfaces.
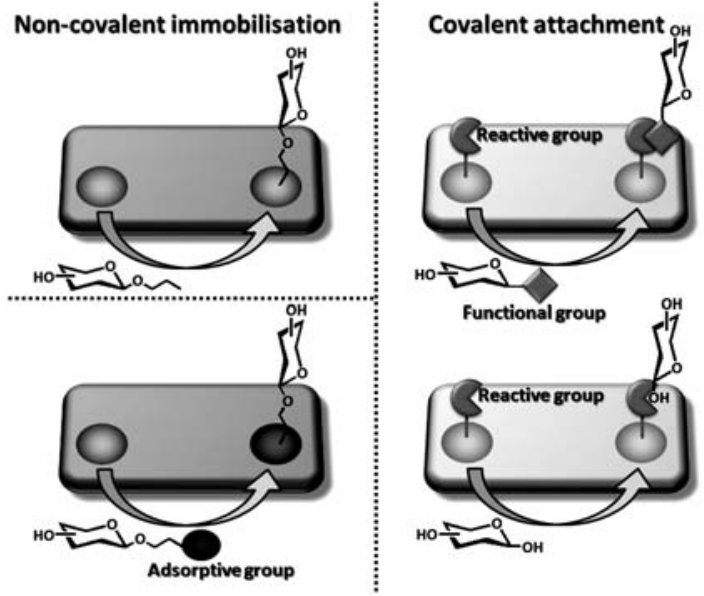

Fig. 9 Immobilisation strategies for carbohydrates on solid surfaces.

Strategies involving non-covalent immobilisation are related to higher flexibility of the macromolecules on the supports and thus with an easier alignment of the binding sites, ${ }^{71}$ although these features are not proven experimentally. Their main advantage is the possibility of a direct use (without any pretreatment or modification) of natural carbohydrates that are simply deposited by physical adsorption on properly treated surfaces. Usually modified microscope glass slides are used as supports. However, other supports such as nitrocellulose, ${ }^{56,58}$ modified polystyrene, ${ }^{57}$ and polyvinylidene fluoride ${ }^{58}$ have been also used as immobilisation platforms in different approaches. The molecular weight of the used polysaccharides should be high enough to guarantee sufficient immobilisation. Hence, oligosaccharides and monosaccharides cannot be densely and stably immobilised at the surface if they are not coupled to another larger moiety such as lipids ${ }^{58,59}$ or fluorous tags $\left(\mathrm{C}_{8} \mathrm{~F}_{17}\right){ }^{61}$ Therefore, strategies involving covalent coupling are preferred for immobilisation of simple carbohydrates and oligosaccharides

Table 4 Methods for attachment of glycans to the surface of microarray

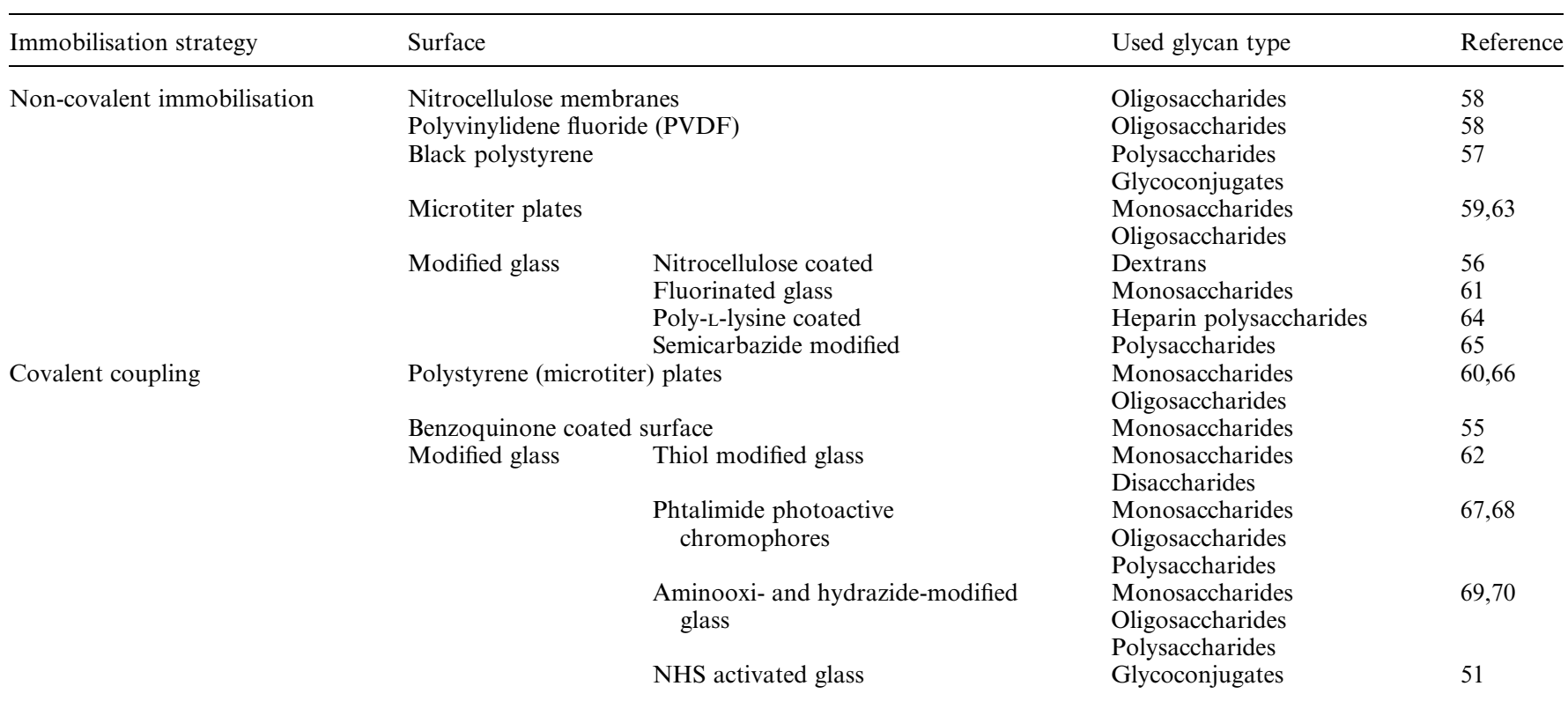


(Fig. 10). Usually, these strategies require both chemically modified glycans and derivatised surfaces. The role of the linkers between the coupled glycan and the support must be stressed out because they govern both binding of the protein and suppression of the non-specific binding. In general, hydrophilic linkers (e.g. PEG) have better binding properties for proteins. Their length also influences the binding affinities: glycans linked by longer tethers interact with proteins stronger than those connected by shorter linkers do. Site-specific, covalent immobilisation of chemically unmodified carbohydrates on modified surfaces (Fig. 10D) is the best option for constructing glycoarrays. This strategy avoids the use of functionalised sugars, which are typically prepared by multistep synthetic methods. A large number ${ }^{58}$ of sugars with different size (monosaccharides, oligosaccharides and polysaccharides) have been immobilised on aminooxy- or hydrazide modified glass surfaces ${ }^{70,72}$ under different conditions (temperature, time, $\mathrm{pH}$ and concentration). It has been observed that carbohydrates immobilised on hydrazide surface exhibit more intense signals after lectin binding than those immobilised on aminooxy-coated slides. An alternative approach that does not require modified sugars involves photo-induced covalent attachment of carbohydrates to surfaces that are coated with photoactive phtalimide derivatives. ${ }^{67,68}$ Upon exposure to light, the grafted surface adsorbs the carbohydrates by hydrogen abstraction followed by radical recombination. The immobilisation yield is much less dependent on the molecular weight of the spotted carbohydrates and in fact, shows higher grafting efficiency for lower molecular weight glycans.
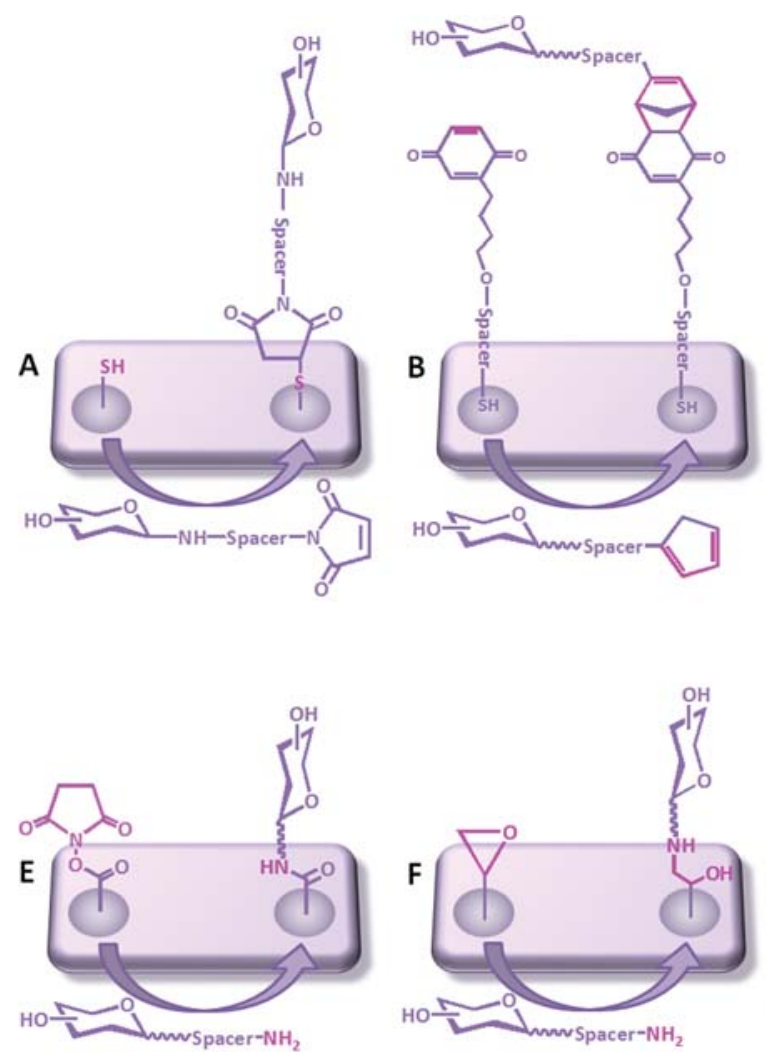
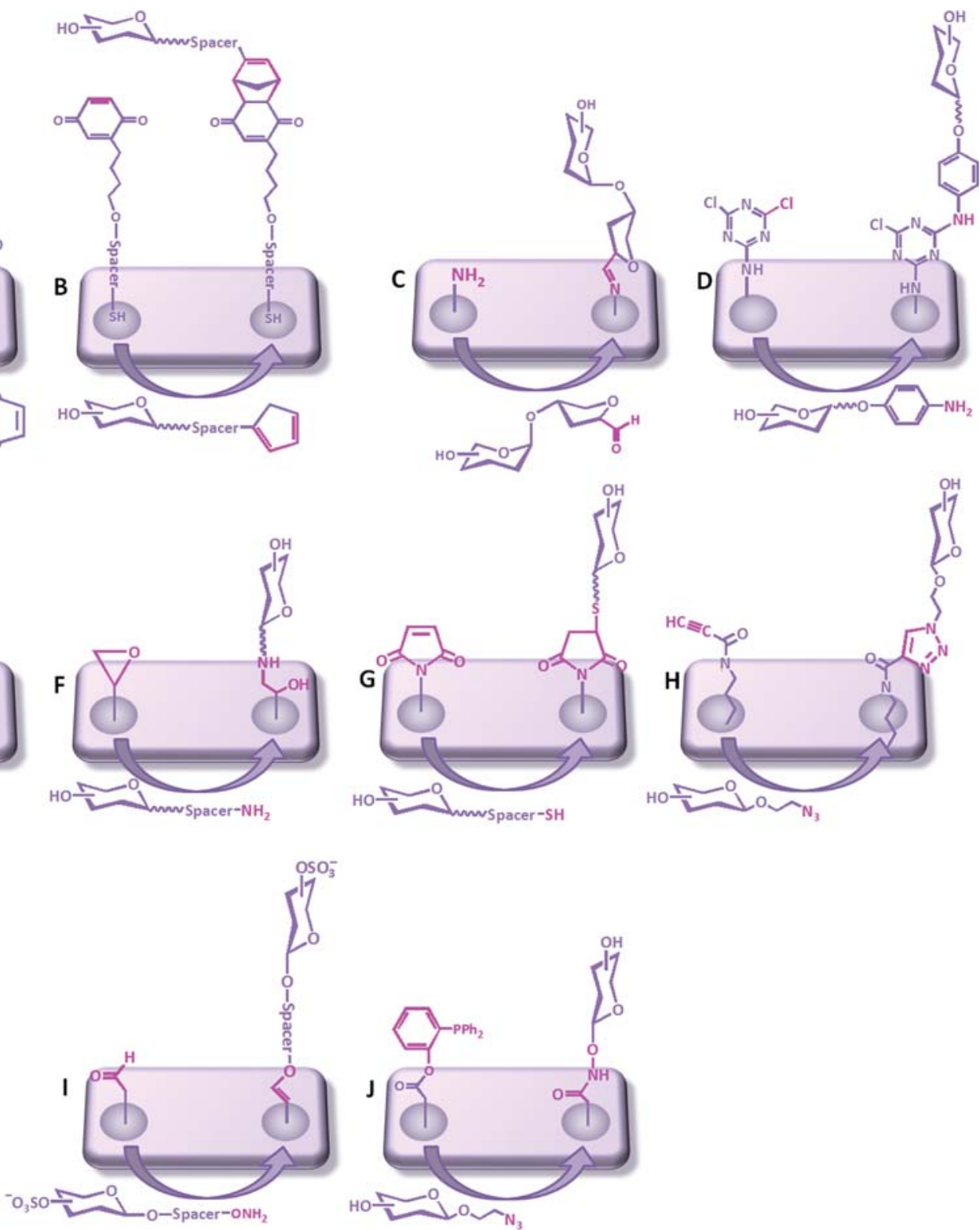

Fig. 10 A schematic presentation of different strategies for covalent, site specific immobilisation of modified glycans on surfaces functionalised with thiol groups (A), benzoquinone (B), amino groups (C), cyanur chloride (D), $N$-hydroxysuccinimide ester (E), epoxy groups (F), maleimide (G), alkyne $(\mathrm{H})$, aldehyde (I) and phosphane (J). 
The detection of the interactions is usually performed by fluorescence where the dye is either directly attached to the analyte or indirectly via a fluorescently marked labelling agent. ${ }^{18}$ Fluorescence-based methods are preferred not only because of their high sensitivity but also because they are compatible with various materials such as glass or gold. However, it must be noted that this procedure often results in protein denaturation or interference with carbohydrate ligand binding. Thus, detection techniques that do not require protein labelling are necessary. Surface plasmon resonance (SPR) spectroscopy ${ }^{55,73-77}$ and mass spectrometry ${ }^{60,63,66,78-81}$ are options but they need to be adapted and optimised to work properly in a high-throughput manner.

In spite of the technological differences mentioned above, glycoarrays share a number of common advantages such as large capacity, high detection sensitivity and long-lasting stability. While the first two advantages are related with the use of microarray platform, the third one is specific for carbohydrates; most of them are stable at room temperature as dry powders. Hence, the arrays produced from them can be kept at room temperature for months or years without structural or immunological changes. ${ }^{57,82}$ These arrays were proposed as an advanced technology just few years ago but they have already demonstrated their high potential in the TE and biomedical fields (Fig. 11). Analysis of carbohydrate-bioentities interactions, profiling of the specific binding of antibodies, elucidation on carbohydrate-mediated cell recognition events, and detection of pathogens for diagnosis are only some examples of important glycoarrays applications.

\section{Manipulation of cell surface glycans: carbohydrates as third generation biomaterials}

Analysis of glycosylation patterns of different cells and tissues has shown that these patterns are controlled but they are not genetically coded. This allows dynamic regulation of the overall structure and shape of sugar molecules and makes the cell flexible and able to respond quickly to change. In fact, our immune system is based on that flexibility: many pathogens have glycanbinding proteins (GBPs) that recognise host glycan structure as receptors for attachment (Table 5). In turn, the eukaryotic

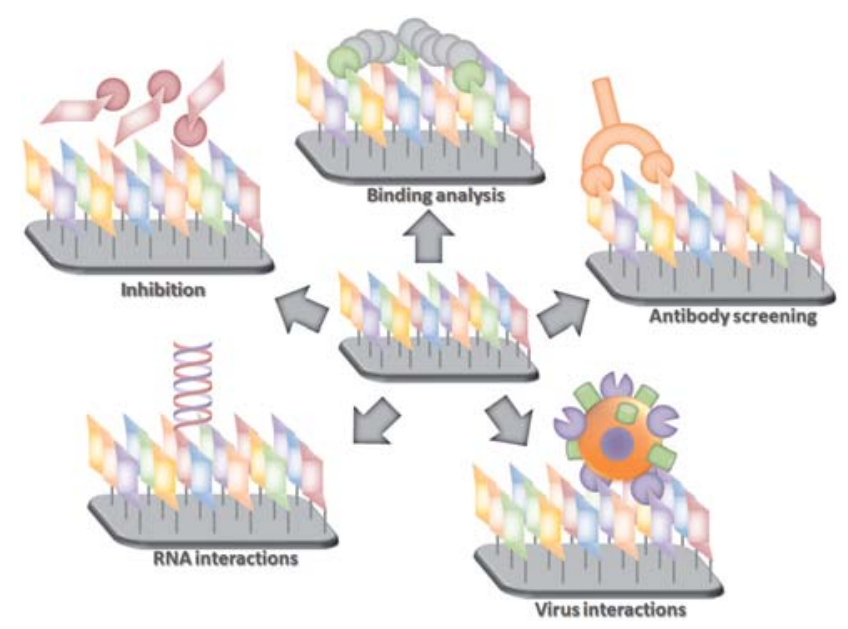

Fig. 11 Several applications of carbohydrate microarrays.
Table 5 Saccharide specificity for some pathogens and toxines. ${ }^{10}$

\begin{tabular}{ll}
\hline Pathogen/Virus & Broad saccharide specificity \\
\hline $\begin{array}{l}\text { Escherichia coli } \\
\text { Type 1 pili }\end{array}$ & \\
P & $\operatorname{Man}(\alpha 1,3) \mathrm{Man}(\alpha 1,6) \mathrm{Man}$ \\
$\mathrm{S}$ & $\mathrm{NeuAc}(\alpha 2,3) \mathrm{Gal}(\beta 1,3) \mathrm{GalNAc}$ \\
CFA/1 & NeuAc $(\alpha 2,8) \mathrm{NeuAc}$ \\
K99 & NeuGc $(\alpha 2,3) \mathrm{Gal}(\beta 1,4) \mathrm{Glc}$ \\
Bordetella pertussis & Gal $(\beta 1,4) \mathrm{Glc}-\mathrm{ceramide}$ \\
Neisseria gonorrheae & Gal $(\beta 1,4) \mathrm{GalNAc}$ \\
Foot and mouth virus & Heparin sulfate \\
Avian influenza virus & 2,3 linked sialic acids \\
Human influenza & 2,6 linked sialic acids \\
Influenza C & $9-O$-acetyl sialic acids \\
Botulinum neurotoxins & gangliosides GD1a, GD1b, GT1a \\
Shiga toxin 1 and 2 & Gal $(\alpha 1,4) \mathrm{Gal}(\beta 1,4) \mathrm{Glc}-\mathrm{ceramide}$ \\
& $(\mathrm{Gb3})$ and analogues \\
Pertussis toxin & Sialic acid, Gal( $(\beta 1,4) \mathrm{Glc}-\mathrm{ceramide}$ \\
& gangliosides \\
Cholera toxin and LT-I & Ganglioside GM1 \\
E. coli enterotoxin, LT-IIa & Gangliosides GD1b, GD1a, GM1 \\
\end{tabular}

organisms have developed GBPs that recognise some pathogenic glycans in the innate immune response. Carbohydrates are also important for the development of tumours because cancer cells alter the expression of glycoproteins at their cell surface in order to evade the immune system. By covering themselves with the right assortment of glycoproteins, the tumour cells can invade other tissues without being identified as aliens.

Unfortunately, in this evolutionary war, the pathogens are still winning. Thus, the necessity for the development of chemical tools that block oligosaccharides and glycoconjugates formation and/or function has emerged. Two main types of inhibitors have been established during the years: ${ }^{16}$ those that block glycoconjugates biosynthesis and those that interfere with glycoconjugates recognition. The first approach is based mainly on the generation of antagonists of the biosynthetic and processing enzymes. The discovery and development of sialidase inhibitors in the fight against the influenza virus is an excellent example that illustrates the applicability of this approach. Sialidase is an exoglycohydrolase that cleaves $\alpha$-ketosidically linked Neu5Ac residues at the end of various glycoconjugates.$^{83}$ Together with M2 ion channel protein and the lectin haemagglutinin, this enzyme decorates the surface of influenza virus A. In a typical process, the virus adheres to the host cell using its haemagglutinin to recognise glycoconjugates that display terminal $\alpha$-sialic acid (Fig. 12) residues. The virus is then exocytosed and fusion occurs. Subsequent synthesis of viral protein and particle

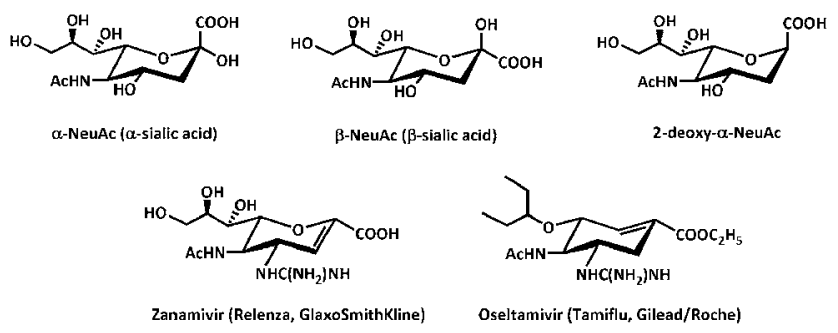

Fig. 12 Chemical structures of $\alpha$ - and $\beta$-anomers of sialic acid and some of the inhibitors developed by mimicking it. 
assembly prepares the virion progeny for the budding process to exit the host cell. Sialidase cleaves the terminal $\alpha$-NeuAc from both the host cell surface and the newly synthesised virion progeny glycoproteins. Thus, the sialidase plays at least two critical roles in the life cycle of the virus: it assists the movement of virus particles through the upper respiratory tract and it helps the release of virion progeny from infected cells.

Initially, sialidase inhibitors have been developed mimicking the structure of $\alpha$-sialic acid. Although 2-deoxy- $\alpha$-D- $N$ acetylneuraminic acid (Fig. 12, 2-deoxy- $\alpha$-NeuAc) did not reach the market, this compound was very important in the studies elucidating the enzymatic mechanism of sialidase. ${ }^{83,84}$ Based on an understanding of the enzyme's catalytic mechanism, the first class drug 4-deoxy-4-guanidino-NeuAc2en (zanamivir) was developed. Zanamivir was launched as an inhalted formulation because of one of the main problems associated with the development of carbohydrates therapeutics: their highly polar nature that turns them into inactive and unstable when applied orally. Thus, the following efforts have been focused toward the development of sialidase inhibitors based on non-carbohydrate templates. Cyclohexenes, ${ }^{85}$ cyclopentanes ${ }^{86}$ and pyrrolidines ${ }^{87}$ have been investigated as core templates. From these trials, the most noteworthy has been the development of the first sialidase inhibitor oseltamivir approved to be used orally. Another effort to target glycosylation enzymes has been proposed by GlycoDesign in the war against cancer. ${ }^{88}$ For several years, clinical trials with swainsonine (GD039) have been ongoing. GD039 is a competitive inhibitor of the Golgi enzyme $\alpha$-mannosidase II $(\alpha \mathrm{MII})$, which is involved in the processing of $N$-linked carbohydrates of newly synthesized glycoproteins as they make their way through the Golgi apparatus to the cell surface (Fig. 3). The $N$-linked pathway mediated by $\alpha$ MII leads to the formation of highly branched carbohydrate structures, which by binding to Lectin-phytohemagglutinin (L-PHA) can be expressed in different types of tumours. When the enzyme $\alpha \mathrm{MII}$ is blocked by swainsonine there is a reduction in L-PHA reactive carbohydrates on cell surfaces; a less aggressive metastatic phenotype; a slower tumour growth; and an accumulation of "hybrid-type" carbohydrates on the cell surface.

Unnatural metabolic substrates are an alternative to the enzyme inhibitors. They are able to intercept the carbohydrate biosynthetic pathways. ${ }^{16,89,90}$ As obvious from Fig. 3, those unnatural metabolic decoys will be active only if they are able to cross the plasma membrane and enter the Golgi where the assembling of the glycoconjugates occurs. Unfortunately, many of the compounds tested so far lack activity in living cells, most probably because their polarity and charge prevents their uptake.

Metabolic interference can generate several responses on the cell surface schematically presented on Fig. 13. A reduction in the amount of the glycoconjugates expressed by cells (Fig. 13A) might occur if the metabolic decoy diverts the oligosaccharides elaboration away from endogenous scaffold destined for the cell surface. ${ }^{89}$ An example for this effect is the use of xylosides as primers. More than 35 years ago, Okayama et al. ${ }^{91}$ found that cells use $\beta$-D-xylopyranosides as primers for glycosaminoglycan formation. Xylosides mimic the natural substrate; they act as acceptors for $\beta$-1,4-galactosyltransferase and thus as primers that divert the assembly process from the endogenous core proteins and cause inhibition of proteoglycan formation. In general, cells

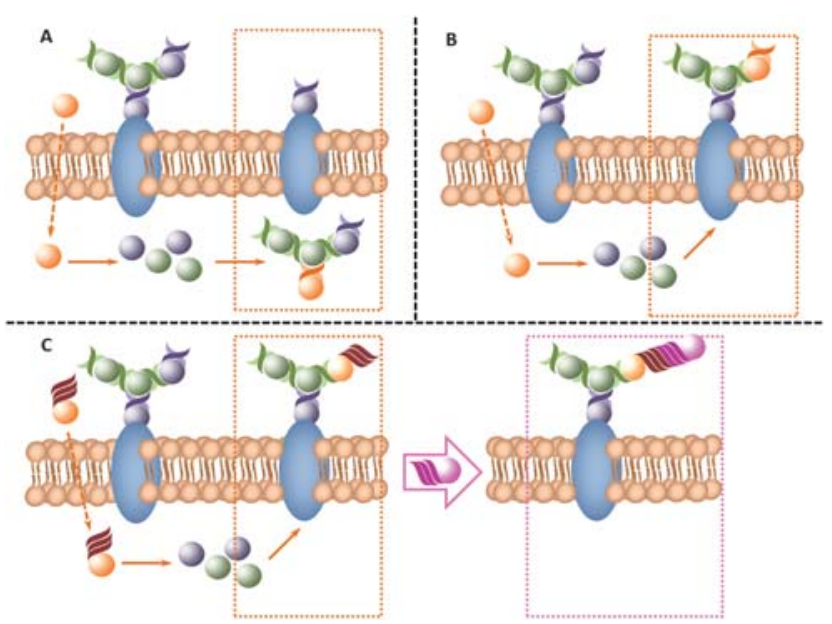

Fig. 13 Modulating cell behaviour via unnatural sugars present in the extracellular space.

incubated with xylosides secrete large amounts of individual glycosaminoglycan chains and accumulate proteoglycans containing truncated chains. The success of $\beta$-D-xylosides in altering proteoglycan biosynthesis suggested that other glycosides might also act as primers (Table 6).

Alternatively, incorporation of unnatural substrates into the cell surface glycoconjugates (Fig. 13B) can occur if the inhibitors are designed to engage the biosynthetic pathway. This approach has been applied in a strategy targeting alteration of the expression of cell surface glycans on tumour cells. ${ }^{92,93}$ Tumour cells express sialic acids that are usually tolerated by the immune system. Tumour immunity can be generated in principle by introducing unnatural sialic acid precursors whose metabolic incorporation leads to the generation of unnatural sialic acid epitopes (Fig. 14). Using this strategy, more immunoreactive cells have been engineered by adjusting the R-group in the mannosamine. ${ }^{93}$ Such unnatural epitopes can be used as vaccines; tumour cells might be preferentially targeted by this approach due to higher expression of sialic acids.

In a third situation, an incorporation of unnatural sugar bearing reactive functional group or marker can lead to the formation of glycan structure that can be further modified by chemical reactions at the cell surface (Fig. 13C). An emerging approach toward glycan visualisation, so-called "bioorthogonal chemical reporter", is based on this response and it has been proposed by Prescher and Bertozzi. ${ }^{94}$ This approach hinges on

Table 6 Glycoside primers with potential use in TE and the biosynthetic pathways affected by them ${ }^{89}$

\begin{tabular}{ll}
\hline Glycoside & Pathway affected \\
\hline Xyl $\beta$-OR & $\begin{array}{l}\text { Glycosaminoglycans and } \\
\text { glycolipids } \\
\text { Glycosaminoglycans } \\
O \text {-glycans found on glycoproteins } \\
\text { and mucins }\end{array}$ \\
Gal $\beta$-OR; Gal $\beta 4$ Xyl-OR & $\begin{array}{l}\text { Polylactosamines } \\
\text { LalNAc } \alpha \text {-OR }\end{array}$ \\
GalNAc $\beta$-OR & Peracetylated Gal $\beta 4$ GlcNAc $\beta$-OR \\
\hline
\end{tabular}




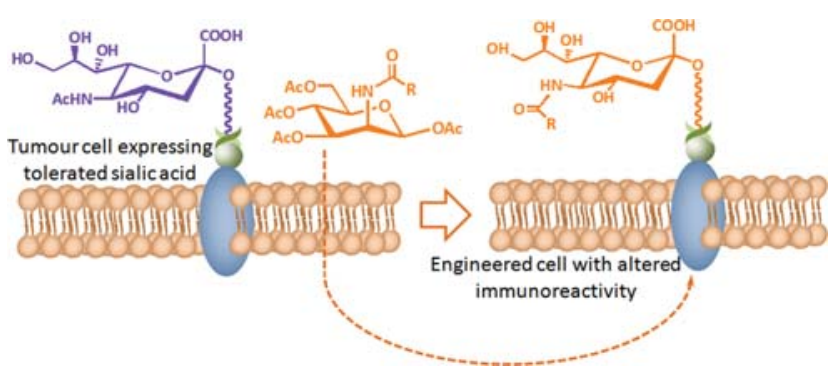

Fig. 14 Boosting tumour reactivity by alternation of cell surface glycans.

the selection of a reporter group that can be readily installed on the monosaccharide substrates with minimal structural perturbation since they must be then processed similarly to their native counterpart and integrated into cellular glycans, i.e. the enzymes involved in the installation process must tolerate the unnatural motif. Hence, the fluorescein probes cannot be used in direct modifications because of their large size. Organic azides have been found to be ideal chemical reporters (Fig. 15); they are small, metabolically stable, and essentially inert in biological systems. Moreover, they fulfil the requirements for the further covalent reaction with a probe bearing chemical moiety (Fig. 13C): the chemical bond must be formed at physiological environment and at the same time to remain stable and inert to the surrounding biological milieu. All these requirements rose from the targeted application: in vivo non-invasive imaging of glycan expression on cell surface. Mice were injected with either ManNAz or GalNAz (Fig. 15) for several days and then
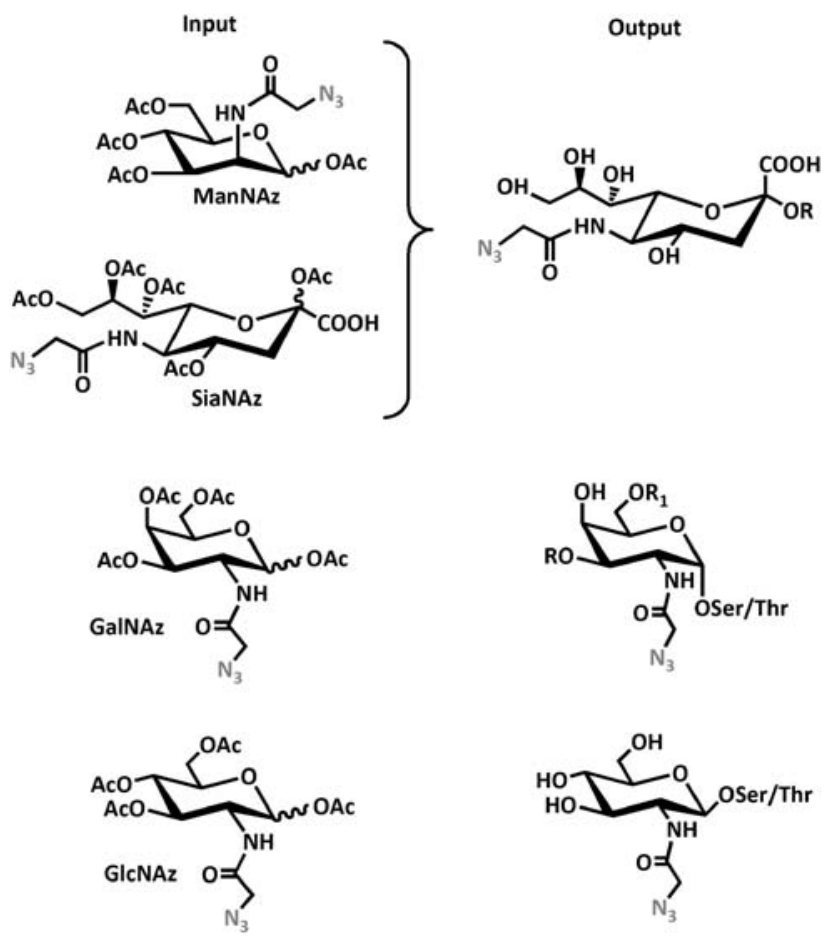

Fig. 15 Several azides are used as bioorthogonal chemical reporters: ${ }^{94}$ azido analogues of natural sugars (left column) are metabolised by cells and converted to cell surface azido sialosides (right column). administered a phosphine probe. ${ }^{95}$ After several hours, the anticipated product was observed on splenocyte cell surfaces and serum glycoproteins.

As already mentioned, the function of surface oligosaccharides and glycoconjugates can be also be altered by interference of glycoconjugates recognition. Two complimentary strategies can be used to achieve this goal: the active centre of the natural carbohydrate receptor can be blocked with a corresponding antagonist or the carbohydrate presented on the cell surface can be blocked by synthetic carbohydrate receptors.

In the quest of identification of different carbohydrate receptor antagonists, the sialyl Lews ${ }^{\mathrm{x}}$ tetrasaccharide (Fig. $16-1$ sLe $^{\mathrm{x}}$ ) became a lead structure. $\mathrm{sLe}^{\mathrm{x}}$ has important roles in regulating the inflammatory processes and in cancer metastasis; it is a common epitope of the physiological selectin ligands and it is recognised by the three known selectins ( $E$-, P- and L-selectin) ${ }^{50,96,97}$ Extensive research has elucidated that the essential pharmacophores required for binding to $E$ - and L-selectins are all three hydroxyl groups of fucose, the 2- and 6- hydroxyl groups of the galactose and the carboxylate of the neuraaminic acid. The binding to P-selectin is similar except that the 2- and 4-hydroxyl groups of fucose are not involved critically.

After the establishment of the $\mathrm{sLe}^{\mathrm{x}}$ functional groups important for complexation, mimics of the tetrasaccharide have been synthesised that are potent selectin antagonists. For example, the compound 3 presented on Fig. 16 is about 5 fold more active at blocking $E$-selectin than sLex. ${ }^{98}$

The design of synthetic carbohydrate receptors has been inspired by the crystal structures of protein-carbohydrate complexes and has seen considerable progress in the past few years. ${ }^{99}$ The main difficulty in the development of artificial carbohydrate receptors arises from the fact that most of the oligosaccharides binding sites are relatively shallow and solvent exposed. Thus, the interactions of a receptor with the hydroxyl groups of a carbohydrate-derived substrate do not fundamentally differ from interaction with water molecules. At least two types of interactions are realised in the described biomimetic sugar receptors: hydrogen bonding and $\mathrm{C}-\mathrm{H} \cdots \pi$ interactions formed by a coplanar arrangement between the substrate and the aromatic receptors subunits. Recent investigation in the Davis group $^{100}$ demonstrated that efficient and selective recognition of

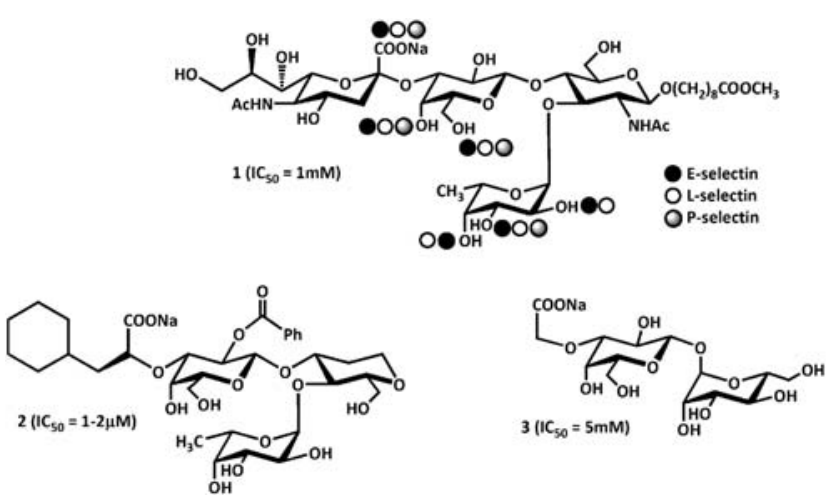

Fig. 16 Sialyl Lewis ${ }^{\mathrm{x}}(1)$ and its functional groups, which are critical for binding to the selectins together with two $E$-selectin antagonists mimicking sLe ${ }^{\mathrm{x}}$ structure $\left(2\right.$ and $\left.3^{98}\right)$. 


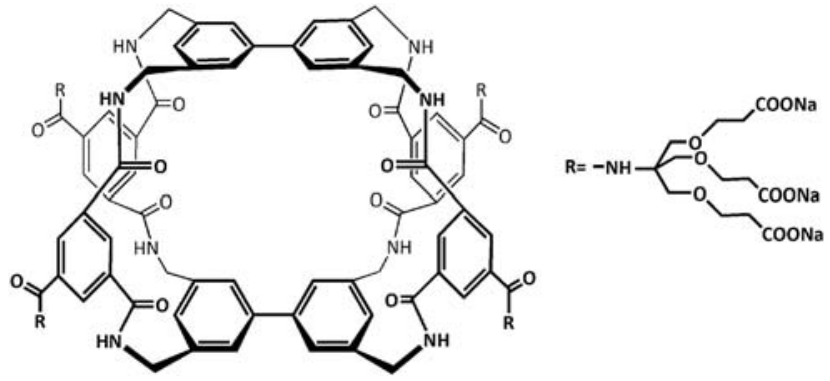

Fig. 17 Biomimetic carbohydrate receptor synthesised by Davis et al. ${ }^{100}$ which demonstrated efficient and selective recognition of carbohydrates in water.

carbohydrates in water can indeed be realised by using the designed by them receptor represented on Fig. 17. An issue, which needs to be addressed further, is whether such receptors exert a characteristic effect on cell adhesion phenomena.

\section{Conclusions}

In the fast developing field of glycomics, scientists seem to have risen to the challenge, making several crucial technological advances in the past few years and overcoming at least some of the obstacles to the elucidation and further exploitation of the "sugar code". Arrays, natural glycan libraries and synthetic chemistry are at the forefront of technology developments and are supported by progress in the development of essential tools for data handling, bioinformatics and molecular modelling of glycans. A major promise of glycoscience is its potential to be used to manipulate cell-bioentity communication and applied in the development of tissue regeneration strategies and treatment of different diseases. These approaches can also lead to progress in the diagnosis and therapeutic products based on specific carbohydrates. Further progress in decoding the glycome must bring new insights into its myriad functions and producing diverse advances in the TE arena. Glycoscience can gain more attention and development in the future only if the glycoscientists are working like the sugars they are studying - they must be highly interactive between themselves in order to function well.

\section{Acknowledgements}

The authors acknowledge the funding from the European Union Seventh Framework Programme (FP7/2007-2013) under grant agreement no. NMP4-SL-2009-229292.

\section{References}

1 R. Langer and J. P. Vacanti, Science, 1993, 260, 920-926.

2 L. L. Hench, Science, 1980, 208, 826-831.

3 L. L. Hench and J. Wilson, Science, 1984, 226, 630-636.

4 L. L. Hench and J. M. Polak, Science, 2002, 295, 1014.

5 S. Hurtley, R. Service and P. Szuromi, Science, 2001, 291, 23372337.

6 T. W. Rademacher, R. B. Parekh and R. A. Dwek, Annu. Rev. Biochem., 1988, 57, 785-838.

7 A. Varki, et al., Essentials of Glycobiology, Cold Spring Harbor Laboratory Press, USA, 2nd edn, 2008, p. 784.

8 T. van der Werff, MIT Tech. Rev., 2003, Feb.

9 D. B. Werz, et al., ACS Chem. Biol., 2007, 2, 685-691.

10 A. Weiss and S. Iyer, Microbe, 2007, 2, 489-497.
11 H.-J. Gabius, Naturwissenschaften, 2000, 87, 108-121.

12 R. A. Laine, Glycobiology, 1994, 4, 759-767.

13 J. S. Bardi, TSRI News and Views, 2001, Oct 8.

14 P. H. Seeberger, Nat. Chem. Biol., 2009, 5, 368-72.

15 A. Varki, Glycobiology, 1993, 3, 97-130.

16 C. R. Bertozzi and L. L. Kiessling, Science, 2001, 291, 2357-2364.

17 N. H. Packer, et al., Proteomics, 2008, 8, 8-20.

18 T. Horlacher and P. H. Seeberger, Chem. Soc. Rev., 2008, 37, 1414 1422.

19 N. Horan, et al., Proc. Natl. Acad. Sci. U. S. A., 1999, 96, 11782 11786.

20 P. H. Seeberger and D. B. Werz, Nature, 2007, 446, 1046-1051.

21 M. F. Murrell, K. J. Yarema and A. Levchenko, ChemBioChem, 2004, 5, 1334-1347.

22 G. Coullerez, P. H. Seeberger and M. Textor, Macromol. Biosci., 2006, 6, 634-647.

23 P. H. Seeberger and W.-C. Haase, Chem. Rev., 2000, 100, 4349-4393.

24 C. C. Wang, et al., Nature, 2007, 446, 896-899.

25 S. Raghavan and D. Kahne, J. Am. Chem. Soc., 1993, 115, 1580 1581.

26 Z. Y. Zhang, et al., J. Am. Chem. Soc., 1999, 121, 734-753.

27 T. Takahashi, et al., Tetrahedron Lett., 2000, 41, 2599-2603.

28 Y. H. Wang, X. S. Ye and L. H. Zhang, Org. Biomol. Chem., 2007, 5, 2189-2200.

29 H. Paulsen, Angew. Chem., Int. Ed. Engl., 1982, 21, 155-173.

30 B. Fraser-Reid, et al., J. Org. Chem., 1990, 55, 6068-6070.

31 S. V. Ley and H. W. M. Priepke, Angew. Chem., Int. Ed. Engl., 1994, 33, 2292-2294.

32 N. L. Douglas, et al., J. Chem. Soc., Perkin Trans. 1, 1998, 51-65.

33 H. Yamada, T. Kato and T. Takahashi, Tetrahedron Lett., 1999, 40, 4581-4584.

34 H. Yamada, et al., Tetrahedron Lett., 1994, 35, 3979-3982.

35 H. Tanaka, et al., Org. Lett., 2002, 4, 4213-4216.

36 X. F. Huang, et al., Angew. Chem., Int. Ed., 2004, 43, 5221-5224.

37 T. J. Boltje, T. Buskas and G. J. Boons, Nat. Chem., 2009, 1, 611622.

38 Z. T. Li and J. C. Gildersleeve, J. Am. Chem. Soc., 2006, 128, 11612 11619.

39 R. Geurtsen and G. J. Boons, Tetrahedron Lett., 2002, 43, 9429 9431.

40 J. D. C. Codee, et al., J. Am. Chem. Soc., 2005, 127, 3767-3773.

41 O. J. Plante, E. R. Palmacci and P. H. Seeberger, Science, 2001, 291, $1523-1527$.

42 P. H. Seeberger, Carbohydr. Res., 2008, 343, 1889-1896.

43 K. M. Koeller and C. H. Wong, Chem. Rev., 2000, 100, 4465-4493. 44 P. Sears and C.-H. Wong, Science, 2001, 291, 2344-2350.

45 G. J. L. Bernardes, B. Castagner and P. H. Seeberger, ACS Chem. Biol., 2009, 4, 703-713.

46 H. Yu, et al., J. Am. Chem. Soc., 2005, 127, 17618-17619.

47 H. Yu, et al., Angew. Chem., Int. Ed., 2006, 45, 3938-3944.

48 L. F. Mackenzie, et al., J. Am. Chem. Soc., 1998, 120, 5583-5584.

49 S. M. Hancock, M. D. Vaughan and S. G. Withers, Curr. Opin. Chem. Biol., 2006, 10, 509-519.

50 P. Sears and C. H. Wong, Proc. Natl. Acad. Sci. U. S. A., 1996, 93, 12086-12093.

51 O. Blixt, et al., Proc. Natl. Acad. Sci. U. S. A., 2004, 101, 17033 17038.

52 M. D. Disney and P. H. Seeberger, Chem. Biol., 2004, 11, 1701-1707.

53 J. C. Paulson, O. Blixt and B. E. Collins, Nat. Chem. Biol., 2006, 2, 238-248.

54 P. R. Crocker and T. Feizi, Curr. Opin. Struct. Biol., 1996, 6, 679691.

55 B. T. Houseman and M. Mrksich, Chem. Biol., 2002, 9, 443-454.

56 D. N. Wang, et al., Nat. Biotechnol., 2002, 20, 275-281.

57 W. G. T. Willats, et al., Proteomics, 2002, 2, 1666-1671.

58 S. Fukui, et al., Nat. Biotechnol., 2002, 20, 1011-1017.

59 M. C. Bryan, et al., Chem. Biol., 2002, 9, 713-720.

60 F. Fazio, et al., J. Am. Chem. Soc., 2002, 124, 14397-14402.

61 K. S. Ko, F. A. Jaipuri and N. L. Pohl, J. Am. Chem. Soc., 2005, 127, $13162-13163$

62 S. J. Park and I. J. Shin, Angew. Chem., Int. Ed., 2002, 41, 3180.

63 F. Fazio, et al., Tetrahedron Lett., 2004, 45, 2689-2692.

64 E. L. Shipp and L. C. Hsieh-Wilson, Chem. Biol., 2007, 14, 195-208.

65 O. Carion, et al., ChemBioChem, 2006, 7, 817-826.

66 M. C. Bryan, et al., J. Am. Chem. Soc., 2004, 126, 8640-8641. 
67 G. T. Carroll, et al., Langmuir, 2006, 22, 2899-2905.

68 G. T. Carroll, et al., Glycoconjugate J., 2008, 25, 5-10.

69 I. Shin, S. Park and M. R. Lee, Chem.-Eur. J., 2005, 11, 2894-2901.

70 S. Park, M. R. Lee and I. Shin, Bioconjugate Chem., 2009, 20, 155162.

71 E. H. Song and N. L. B. Pohl, Curr. Opin. Chem. Biol., 2009, 13, 626632.

72 M. Lee and I. Shin, Org. Lett., 2005, 7, 4269-4272.

73 E. A. Smith, et al., J. Am. Chem. Soc., 2003, 125, 6140-6148.

74 A. R. de Boer, et al., Glycoconjugate J., 2008, 25, 75-84.

75 C. F. Grant, et al., Langmuir, 2008, 24, 14125-14132.

76 R. Karamanska, et al., Glycoconjugate J., 2008, 25, 69-74.

77 G. M. Hwang, et al., IEEE Sens. J., 2008, 8, 2074-2079.

$78 \mathrm{~J} . \mathrm{Su}$ and M. Mrksich, Angew. Chem., Int. Ed., 2002, 41, 4715-4718.

79 Z. L. Zhi, et al., ChemBioChem, 2008, 9, 1568-1575.

80 N. Laurent, et al., ChemBioChem, 2008, 9, 883-887.

81 A. Kameyama, Trends Glycosci. Glycotechnol., 2006, 18, 323-341.

82 D. N. Wang, Proteomics, 2003, 3, 2167-2175.

83 M. von Itzstein, Nat. Rev. Drug Discovery, 2007, 6, 967-974.

84 A. K. J. Chong, et al., Eur. J. Biochem., 1992, 207, 335-343.
85 C. U. Kim, et al., J. Am. Chem. Soc., 1997, 119, 681-690.

86 Y. S. Babu, et al., J. Med. Chem., 2000, 43, 3482-3486.

87 D. A. DeGoey, et al., J. Org. Chem., 2002, 67, 5445-5453.

88 P. E. Shaheen, et al., Invest. New Drugs, 2005, 23, 577-581.

89 J. R. Brown, M. M. Fuster and J. D. Esko, Trends Glycosci. Glycotechnol., 2001, 13, 335-343.

90 J. A. Prescher and C. R. Bertozzi, Cell, 2006, 126, 851-854

91 M. Okayama, K. Kimata and S. Suzuki, J. Biochem., 1973, 74, 1069 1073.

92 M. M. Fuster and J. D. Esko, Nat. Rev. Cancer, 2005, 5, 526-542.

93 G. A. Lemieux and C. R. Bertozzi, Chem. Biol., 2001, 8, 265-275.

94 J. A. Prescher and C. R. Bertozzi, Nat. Chem. Biol., 2005, 1, 13-21.

95 J. A. Prescher, D. H. Dube and C. R. Bertozzi, Nature, 2004, 430, 873-877.

96 E. E. Simanek, et al., Chem. Rev., 1998, 98, 833-862.

97 G. Thoma, et al., J. Med. Chem., 1999, 42, 4909-4913.

98 P. Sears and C. H. Wong, Angew. Chem., Int. Ed., 1999, 38, 23012324.

99 S. Kubik, Angew. Chem., Int. Ed., 2009, 48, 1722-1725.

100 Y. Ferrand, et al., Angew. Chem., Int. Ed., 2009, 48, 1775-1779. 